\title{
Integrated Spectra of Milky Way Globular Clusters
}

 \\ ${ }^{1}$ Universidade de São Paulo, IAG, Rua do Matão 1226, Cidade Universitária, São Paulo 05508-900, Brazil \\ ${ }^{2}$ Universidade Federal do Rio Grande do Sul, Departamento de Astronomia, CP 15051, Porto Alegre 91501-970, Brazil \\ Received 2019 July 16; revised 2019 August 20; accepted 2019 August 26; published 2019 October 25
}

\begin{abstract}
Integrated spectra of Milky Way globular clusters (GCs) are reproduced by computing synthetic spectra taking into account individual element abundances. Five clusters were selected from their location in the Galactic bulge, for which integrated spectra were available in the WiFeS Atlas of Galactic Globular Cluster Spectra project. Our aim is to further study the oldest GCs located in the Galactic bulge, with a metallicity in the range $-1.6<$ $[\mathrm{Fe} / \mathrm{H}]<-0.7$. We also include the halo cluster NGC 6752 for comparison purposes. We reproduce the full spectra in the range 4500-9000 $\AA$ available in these observed spectra, as well as individual lines of $\mathrm{Na}, \mathrm{Mg}, \mathrm{Al}, \mathrm{Si}$, $\mathrm{Ca}, \mathrm{Ti}, \mathrm{Ba}$, and $\mathrm{Eu}$. We report a list of lines that are suitable for abundance derivation, and by adopting these abundances, we are able to fit the damping parameters that define the wings of strong lines of well-known triplets of Mg I and Ca II. Finally, the effect of multiple stellar populations through enhanced $\mathrm{Na}$ abundances is tested.
\end{abstract}

Unified Astronomy Thesaurus concepts: Galactic bulge (2041); Globular star clusters (656); Galaxy abundances (574); Stellar atmospheres (1584); Stellar populations (1622)

\section{Introduction}

Integrated spectra of Milky Way (MW) globular clusters (GCs) are becoming an increasingly important ingredient in the study of faint and compact GCs in our Galaxy and in other galaxies, as well as the integrated spectra of galaxies.

The integrated spectra of MW GCs observed by Bica \& Alloin (1986), Schiavon et al. (2005), and Usher et al. (2017) offer an important basis for the validation of codes that try to reproduce integrated spectra of star clusters and old galaxies.

At the same time, given the degeneracy between metallicity and age, in order to reproduce the integrated spectra, it is best to have input data on ages and chemical abundances, which is possible for MW and Magellanic Cloud GCs. Compilations of such data for the MW were presented by Pritzl et al. (2005) and Harris (1996, 2010 edition). ${ }^{3}$ In a series of papers, Saviane et al. (2012), Dias et al. (2015, 2016), and Vásquez et al. (2018) derived abundances and alpha-to-iron ratios from FORS2@VLT spectra for individual stars in $61 \mathrm{GCs}$, targeting less wellstudied ones, and compared them with the literature.

Work on derivation of abundances from integrated spectra is becoming available for the old GCs in the Galaxy, where ages from the literature are adopted. Roediger et al. (2014) derived abundances from the integrated spectra of 41 GCs for which Schiavon et al. (2005) had obtained the integrated spectra. Sakari et al. (2013) and Colucci et al. (2017, and references therein) were able to measure equivalent widths (EWs) and derive abundances. Larsen et al. (2017, and references therein) and Conroy et al. (2018, and references therein) were able to derive metallicities and abundances from full spectrum fitting of the integrated spectra of GCs.

A recent review on the building of integrated spectra for single stellar populations (SSPs) is presented by Martins et al. (2019). They investigate the methods and ingredients used, including differences between evolutionary tracks or use of observed color-magnitude diagrams (CMDs), initial mass

\footnotetext{
www.physics.mcmaster.ca/ harris/mwgc.dat
}

functions (IMFs), and spectral libraries available in the literature.

In the present work, we use spectra from the WiFeS Atlas of Galactic Globular Cluster Spectra (WAGGS) project by Usher et al. (2017), that made available integrated spectra of a series of GCs. We have selected for our analysis, six GCs from Usher et al. (2017) that have good signal-to-noise ratios (S/Ns), where five of them are contained in the list of 43 bulge GCs as selected by Bica et al. (2016), and additionally, the halo cluster NGC 6752, for comparisons with the literature. We also limited the metallicity to be within $-1.6<[\mathrm{Fe} / \mathrm{H}]<-0.7$, in order to concentrate efforts on the oldest GCs of the Galactic bulge.

For the building of the integrated spectra, we here describe in detail the code SynSSP. In this code we adopt the Dartmouth (Dotter et al. 2008) isochrones, and compute the spectra of a series of stars along the main-sequence (MS), subgiant, and red giant phases, for a given age, metallicity, $[\alpha / \mathrm{Fe}]$, and element abundances. The synthetic spectra are computed in each run. A previous version of this procedure was already described in $\mathrm{La}$ Barbera et al. (2013). The code is now much improved, mainly due to the update of the code PFANT for spectrum synthesis, as described in Barbuy et al. (2018b). The code was entirely upgraded with homogeneization of language to Fortran 2003, optimization for speed, and improved error reporting in case of problems with the input data files. Line lists were also revised.

The ages are adopted from the recent age derivations by Kerber et al. (2018), R. A. P. Oliveira et al. (2019, in preparation), and VandenBerg et al. (2013), from isochrone fitting to CMDs. Our main interest is to reproduce the integrated spectra by computing stellar spectra taking into account their elemental abundances, reported in high-resolution spectroscopic studies, and by further deriving element abundances from the integrated spectra.

We also investigate the multiple stellar populations, in proportions derived by Milone et al. (2017) and R. A. P. Oliveira et al. (2019, in preparation), by analyzing the effect of a combined spectrum of first and second generations, with different $\mathrm{Na}$ abundances. The use of $\mathrm{Na}$ and $\mathrm{TiO}$ line intensities to deduce a bottom-heavy stellar IMF is also discussed. 
Table 1

Galactic Coordinates, Distances to the Sun and to the Galactic Center, Absolute Visual Magnitude from Harris (1996), Fraction of First Generation Stars from Milone et al. (2017), Ages from R. A. P. Oliveira et al. (2019, in preparation) for NGC 6624, NGC 6637, and NGC 6723, Ages from Kerber et al. (2018) for NGC 6522, and Ages from VandenBerg et al. (2013) for NGC 6171 and NGC 6752

\begin{tabular}{|c|c|c|c|c|c|c|c|c|}
\hline Cluster & $l\left(^{\circ}\right)$ & $b\left(^{\circ}\right)$ & $\begin{array}{c}d_{\odot} \\
(\mathrm{kpc})\end{array}$ & $\begin{array}{c}d_{\mathrm{GC}} \\
(\mathrm{kpc})\end{array}$ & $M_{V}$ & $N_{1} / N_{\text {TOT }}$ & $\begin{array}{l}\text { Age } \\
\text { (Gyr) }\end{array}$ & $\delta Y_{1 \mathrm{G}, 2 \mathrm{G}}$ \\
\hline NGC 6171 & 3.37 & 23.01 & 6.4 & 3.3 & -7.12 & 0.397 & 12.0 & 0.019 \\
\hline NGC 6522 & 1.02 & -3.93 & 7.7 & 0.6 & -7.95 & 0.160 & 13.0 & $\ldots$ \\
\hline NGC 6637 & 1.72 & -10.27 & 8.8 & 1.7 & -7.64 & 0.425 & 12.4 & 0.004 \\
\hline NGC 6723 & 0.07 & -17.30 & 8.7 & 2.6 & -7.83 & 0.363 & 12.5 & 0.005 \\
\hline NGC 6752 & 336.49 & -25.63 & 4.0 & 5.2 & -7.73 & 0.294 & 12.5 & $0.010,0.032$ \\
\hline
\end{tabular}

Note. $Y$ are helium enhancements in second generation stars (third for the case of NGC 6752) from Milone et al. (2018, 2019).

Table 2

Literature Metallicities and Abundances for the Sample Clusters

\begin{tabular}{|c|c|c|c|c|c|c|c|c|c|c|c|c|}
\hline Cluster & {$[\mathrm{Fe} / \mathrm{H}]$} & {$[\mathrm{O} / \mathrm{Fe}]$} & {$[\mathrm{Na} / \mathrm{Fe}]$} & {$[\mathrm{Al} / \mathrm{Fe}]$} & {$[\mathrm{Mg} / \mathrm{Fe}]$} & {$[\mathrm{Si} / \mathrm{Fe}]$} & {$[\mathrm{Ca} / \mathrm{Fe}]$} & {$[\mathrm{Ti} / \mathrm{Fe}]$} & {$[\mathrm{Ba} / \mathrm{Fe}]$} & {$[\mathrm{Eu} / \mathrm{Fe}]$} & Method & References \\
\hline \multirow[t]{3}{*}{ NGC 6171} & -1.02 & +0.17 & +0.37 & $\ldots$ & +0.51 & +0.54 & +0.06 & +0.40 & $\ldots$ & $\ldots$ & comp. & Roediger et al. (2014) \\
\hline & -1.02 & $\ldots$ & $\ldots$ & $\ldots$ & +0.41 & +0.25 & +0.32 & +0.42 & $\cdots$ & $\ldots$ & ISFF & Conroy et al. (2018) \\
\hline & -1.58 & $\cdots$ & $\cdots$ & $\cdots$ & $\cdots$ & $\cdots$ & +0.24 & $\cdots$ & $\cdots$ & $\cdots$ & ISEW & Usher et al. (2019) \\
\hline \multirow[t]{2}{*}{ NGC 6522} & -0.95 & +0.36 & +0.05 & +0.20 & +0.23 & +0.13 & +0.13 & +0.04 & +0.32 & +0.30 & HRS & Barbuy et al. (2014) \\
\hline & -1.12 & $\cdots$ & +0.24 & +0.62 & +0.42 & +0.28 & +0.35 & +0.38 & +0.37 & +0.43 & HRS & Ness et al. (2014) \\
\hline \multirow[t]{4}{*}{$(2 \mathrm{G})$} & -1.04 & +0.33 & $\cdots$ & +0.57 & +0.13 & +0.30 & $\cdots$ & $\ldots$ & $\cdots$ & $\cdots$ & HRS & $\begin{array}{l}\text { Fernández-Trincado } \\
\text { et al. (2019) }\end{array}$ \\
\hline & -1.21 & $\ldots$ & $\cdots$ & $\cdots$ & +0.47 & +0.30 & +0.35 & +0.40 & $\cdots$ & $\cdots$ & ISFF & Conroy et al. (2018) \\
\hline & -1.58 & $\cdots$ & $\cdots$ & $\ldots$ & $\ldots$ & $\ldots$ & +0.13 & $\ldots$ & $\ldots$ & $\ldots$ & ISEW & Usher et al. (2019) \\
\hline & -1.34 & +0.49 & +0.04 & $\cdots$ & +0.27 & +0.25 & +0.17 & +0.16 & $\cdots$ & $\cdots$ & comp. & Roediger et al. (2014) \\
\hline \multirow[t]{4}{*}{ NGC 6624} & -0.69 & +0.41 & $\ldots$ & +0.39 & +0.42 & +0.38 & +0.40 & +0.37 & $\cdots$ & $\ldots$ & HRS & Valenti et al. (2011) \\
\hline & -0.77 & $\cdots$ & $\cdots$ & $\cdots$ & +0.36 & +0.25 & +0.23 & +0.41 & $\cdots$ & $\cdots$ & ISFF & Conroy et al. (2018) \\
\hline & -1.58 & $\cdots$ & $\cdots$ & $\cdots$ & $\cdots$ & $\cdots$ & +0.18 & $\cdots$ & $\cdots$ & $\cdots$ & ISEW & Usher et al. (2019) \\
\hline & -0.44 & +0.41 & $\cdots$ & $\cdots$ & +0.42 & +0.38 & +0.40 & +0.37 & $\cdots$ & $\cdots$ & comp. & Roediger et al. (2014) \\
\hline \multirow[t]{4}{*}{ NGC 6637} & -0.77 & +0.20 & +0.35 & +0.49 & +0.28 & +0.45 & +0.20 & +0.24 & +0.22 & +0.45 & HRS & Lee (2007) \\
\hline & -0.90 & $\cdots$ & $\cdots$ & $\cdots$ & +0.43 & +0.20 & +0.30 & +0.54 & $\cdots$ & $\cdots$ & ISFF & Conroy et al. (2018) \\
\hline & -1.58 & $\cdots$ & $\cdots$ & $\cdots$ & $\cdots$ & $\cdots$ & +0.20 & $\cdots$ & $\cdots$ & $\cdots$ & ISEW & Usher et al. (2019) \\
\hline & -0.64 & +0.20 & +0.35 & $\cdots$ & +0.28 & +0.45 & +0.20 & $\cdots$ & $\cdots$ & $\cdots$ & comp. & Roediger et al. (2014) \\
\hline \multirow[t]{3}{*}{ NGC 6723} & -0.98 & +0.29 & +0.00 & 0.31 & +0.23 & +0.36 & +0.30 & +0.24 & +0.22 & - & HRS & $\begin{array}{l}\text { Rojas-Arriagada et al. } \\
\text { (2016) }\end{array}$ \\
\hline & -1.22 & +0.39 & +0.05 & $\cdots$ & +0.58 & $\cdots$ & $\cdots$ & $\cdots$ & $\cdots$ & $\cdots$ & HRS & $\begin{array}{l}\text { Gratton et al. } \\
\text { (2015) (BHB) }\end{array}$ \\
\hline & -1.22 & +0.55 & +0.11 & $\cdots$ & +0.50 & +0.59 & +0.81 & $\cdots$ & +0.75 & $\cdots$ & HRS & $\begin{array}{l}\text { Gratton et al. } \\
\text { (2015) (RHB) }\end{array}$ \\
\hline \multirow[t]{4}{*}{$(1 \mathrm{G})$} & -0.91 & +0.39 & +0.14 & +0.32 & +0.47 & +0.52 & +0.37 & +0.34 & +0.36 & +0.38 & HRS & Crestani et al. (2019) \\
\hline & -1.32 & $\cdots$ & $\cdots$ & $\cdots$ & +0.53 & +0.20 & +0.33 & +0.29 & $\cdots$ & $\cdots$ & ISFF & Conroy et al. (2018) \\
\hline & -1.58 & $\cdots$ & $\cdots$ & $\cdots$ & $\cdots$ & $\ldots$ & +0.49 & $\cdots$ & $\cdots$ & $\cdots$ & ISEW & Usher et al. (2019) \\
\hline & -1.10 & $\cdots$ & $\cdots$ & $\cdots$ & +0.44 & +0.68 & +0.33 & +0.24 & $\cdots$ & $\cdots$ & comp. & Roediger et al. (2014) \\
\hline NGC 6752 & -1.56 & +0.16 & +0.33 & +0.41 & +0.50 & +0.38 & $\cdots$ & $\cdots$ & $\cdots$ & $\cdots$ & HRS & Carretta et al. (2009) \\
\hline (1G) & -1.56 & +0.54 & +0.01 & +0.11 & +0.45 & +0.51 & $\ldots$ & $\cdots$ & $\cdots$ & $\cdots$ & HRS & Carretta et al. (2012) \\
\hline$(2 \mathrm{G})$ & -1.56 & +0.25 & +0.36 & +0.74 & +0.42 & +0.48 & $\ldots$ & $\cdots$ & $\cdots$ & $\cdots$ & HRS & Carretta et al. (2012) \\
\hline \multirow[t]{5}{*}{$(3 G)$} & -1.56 & +0.06 & +0.62 & +1.21 & +0.31 & +0.43 & $\cdots$ & $\cdots$ & $\ldots$ & $\ldots$ & HRS & Carretta et al. (2012) \\
\hline & -1.58 & $\cdots$ & +0.09 & $\cdots$ & $\cdots$ & $\cdots$ & +0.46 & +0.36 & +0.10 & $\cdots$ & ISEW & Colucci et al. (2017) \\
\hline & -1.58 & $\cdots$ & $\cdots$ & $\cdots$ & $\cdots$ & $\cdots$ & +0.26 & $\cdots$ & $\cdots$ & $\cdots$ & ISEW & Usher et al. (2019) \\
\hline & -1.883 & $\cdots$ & +0.302 & $\cdots$ & +0.391 & $\cdots$ & +0.355 & +0.342 & +0.182 & $\cdots$ & ISFF & Larsen et al. (2017) \\
\hline & -1.64 & $\ldots$ & $\ldots$ & $\cdots$ & +0.54 & +0.42 & +0.33 & +0.29 & $\ldots$ & $\cdots$ & ISFF & Conroy et al. (2018) \\
\hline
\end{tabular}

In Section 2, the available literature on the sample clusters is reported. In Section 3, we describe the calculations of integrated spectra of simple stellar populations employed in this work. In Section 4. we examine individual abundances that can be derived from the integrated spectra. In Section 5, the effect of multiple stellar populations on $\mathrm{Na}$ lines is investigated. In Section 6, conclusions are drawn.

\section{Sample GCs}

Our sample is composed of five GCs in the Galactic bulge and one halo cluster in the metallicity range $-1.6 \lesssim[\mathrm{Fe} / \mathrm{H}] \lesssim$ -0.7 , with ages older than $11 \mathrm{Gyr}$, and for which there are available integrated spectra in the WiFeS Atlas of Galactic Globular Cluster Spectra-WAGGS project from Usher et al. (2017). The integrated spectra from the WAGGS library were 
Table 3

List of Lines Inspected in the Integrated Spectra, and Resulting Abundances

\begin{tabular}{|c|c|c|c|c|c|c|c|c|c|}
\hline Species & $\lambda(\AA)$ & $\chi_{\mathrm{ex}}(\mathrm{eV})$ & $\log g f$ & NGC 6171 & NGC 6522 & NGC 6624 & NGC 6637 & NGC 6723 & NGC 6752 \\
\hline $\mathrm{Na} \mathrm{I}$ & 5682.633 & 2.10 & -0.71 & $0.00 \pm 0.15$ & 0.00 & 0.20 & 0.00 & $\ldots$ & 0.00 \\
\hline $\mathrm{Na} \mathrm{I}$ & 5688.194 & 2.11 & -1.40 & $0.35 \pm 0.15$ & 0.35 & 0.35 & 0.35 & 0.35 & 0.35 \\
\hline $\mathrm{Na} \mathrm{I}$ & 5688.205 & 2.11 & -0.45 & $0.35 \pm 0.15$ & 0.35 & 0.35 & 0.35 & 0.35 & 0.35 \\
\hline $\mathrm{Na} \mathrm{I}$ & 6154.230 & 2.10 & -1.56 & $0.35 \pm 0.15$ & 0.20 & 0.35 & 0.35 & 0.35 & $\cdots$ \\
\hline $\mathrm{Na} \mathrm{I}$ & 6160.753 & 2.10 & -1.26 & $0.00 \pm 0.15$ & 0.00 & 0.35 & 0.00 & 0.35 & 0.35 \\
\hline $\mathrm{Na} \mathrm{I}$ & 8183.256 & 2.10 & -0.47 & $0.35 \pm 0.15$ & 0.20 & 0.00 & 0.00 & 0.20 & 0.20 \\
\hline $\mathrm{Na} \mathrm{I}$ & 8194.790 & 2.10 & +0.24 & $0.20 \pm 0.15$ & 0.20 & 0.20 & 0.00 & 0.00 & 0.35 \\
\hline Mg I & 5528.405 & 4.34 & -0.547 & $0.51 \pm 0.15$ & 0.23 & 0.42 & 0.28 & 0.23 & 0.38 \\
\hline Mg I & 5711.088 & 4.34 & -1.842 & $0.51 \pm 0.10$ & 0.23 & 0.42 & 0.28 & 0.45 & 0.25 \\
\hline Al I & 6696.185 & 4.02 & -1.58 & $0.25 \pm 0.20$ & 0.20 & 0.30 & 0.20 & 0.31 & 0.10 \\
\hline Al I & 6696.204 & 4.02 & -1.58 & $0.25 \pm 0.20$ & 0.20 & 0.30 & 0.20 & 0.31 & 0.10 \\
\hline Al I & 6696.788 & 4.02 & -1.42 & $0.25 \pm 0.20$ & 0.20 & 0.30 & 0.20 & 0.31 & 0.10 \\
\hline Al I & 6698.673 & 3.14 & -1.65 & $0.25 \pm 0.20$ & 0.20 & 0.30 & 0.20 & 0.31 & 0.10 \\
\hline Si I & 6414.99 & 5.87 & -1.13 & $0.47 \pm 0.20$ & -0.05 & 0.15 & 0.45 & -0.18 & 0.30 \\
\hline Si I & 7405.79 & 5.61 & -0.66 & $0.05 \pm 0.20$ & -0.50 & 0.08 & 0.05 & 0.20 & 0.47 \\
\hline Si I & 7415.96 & 5.61 & -0.73 & $0.15 \pm 0.20$ & 0.20 & 0.38 & 0.25 & 0.20 & 0.47 \\
\hline Si I & 7423.51 & 5.62 & -0.58 & $0.05 \pm 0.20$ & -0.18 & 0.08 & 0.05 & -0.05 & 0.0 \\
\hline $\mathrm{Ca} \mathrm{I}$ & 6161.295 & 2.51 & -1.02 & $0.21 \pm 0.15$ & 0.13 & 0.45 & 0.40 & 0.53 & 0.20 \\
\hline $\mathrm{Ca} \mathrm{I}$ & 6162.167 & 1.89 & -0.09 & $0.21 \pm 0.15$ & 0.13 & 0.45 & 0.40 & 0.53 & 0.20 \\
\hline $\mathrm{Ca} \mathrm{I}$ & 6439.080 & 2.52 & +0.3 & $0.21 \pm 0.15$ & 0.13 & 0.45 & 0.40 & 0.53 & 0.20 \\
\hline Ti I & 5965.825 & 1.88 & -0.42 & $-0.04 \pm 0.10$ & 0.04 & 0.37 & 0.24 & 0.24 & 0.20 \\
\hline Ti I & 6261.106 & 1.43 & -0.48 & $-0.04 \pm 0.10$ & 0.10 & 0.37 & 0.24 & 0.50 & 0.20 \\
\hline Ti I & 6336.113 & 1.44 & -1.74 & $-0.04 \pm 0.10$ & 0.04 & 0.37 & 0.24 & 0.24 & 0.20 \\
\hline Ba II & 6496.90 & 0.604 & -0.32 & $0.00 \pm 0.15$ & 0.02 & 0.10 & 0.22 & 0.22 & 0.00 \\
\hline Eu II & 6645.064 & 1.38 & +0.12 & $0.50 \pm 0.15$ & 0.55 & 0.00 & 0.45 & 0.00 & 0.50 \\
\hline
\end{tabular}

Note. Uncertainties given for NGC 6171 are the same for all clusters for each respective line.

Table 4

Atomic Constants Adopted for the Mg I and Ca II Triplet Lines

\begin{tabular}{|c|c|c|c|c|c|c|}
\hline Species & $\lambda(\AA)$ & $\chi_{\mathrm{ex}}(\mathrm{eV})$ & $\log g f$ & $C_{6}$ & $\gamma_{R} / \mathrm{NH}$ & $\gamma_{e} / \mathrm{NH}$ \\
\hline $\mathrm{Mg} \mathrm{I}$ & 5167.3216 & 2.7091 & $-0.854(1)$ & $0.3 E-29$ & $1.0 \mathrm{E}+08$ & $2.0 \mathrm{E}-07$ \\
\hline Mg I & 5172.684 & 2.7116 & $-0.363(1)$ & idem & idem & idem \\
\hline Mg I & 5183.604 & 2.7166 & $-0.168(1)$ & idem & idem & idem \\
\hline Ca II & 8498.023 & 1.6924 & $-1.312(2)$ & $0.9 \mathrm{E}-32$ & $3.6 \mathrm{E}+08$ & $2.6 \mathrm{E}-07$ \\
\hline Ca II & 8542.091 & 1.6999 & $-0.362(2)$ & $0.8 \mathrm{E}-32$ & $3.0 \mathrm{E}+08$ & idem \\
\hline $\mathrm{Ca}$ II & 8662.141 & 1.6924 & $-0.623(2)$ & $0.8 \mathrm{E}-32$ & $2.95 \mathrm{E}+08$ & idem \\
\hline
\end{tabular}

References. (1) Pehlivan Rhodin et al. (2017), (2) Kurúcz (2005).

observed with the WiFeS integral field spectrograph installed in the Australian National University $2.3 \mathrm{~m}$ telescope and cover a wide wavelength range from 3300-9050 $\AA$ at a spectral resolution of $R \sim 6800$.

Basic information on the selected GCs, including the galactic coordinates, distances to the Sun and to the Galactic center, and absolute magnitudes $M_{V}$ from Harris (1996) are given in Table 1. In this table, the fractions of first generation (1G) stellar populations relative to the total, as estimated by Milone et al. (2017) from the Hubble Space Telescope ultraviolet filters, and ages deduced from Kerber et al. (2018), R. A. P. Oliveira et al. (2019, in preparation), and VandenBerg et al. (2013) are also given. Finally, He enhancements from a second generation (2G) relative to $1 \mathrm{G}$ from Milone et al. (2019) are listed. Literature chemical abundances derived with different methods are reported in Table 2.

\section{Integrated Spectrum Model}

In order to reproduce the integrated spectrum of a stellar population, the following ingredients are required: stellar evolutionary tracks and a library of stellar spectra. The isochrones determine the mass and the atmospheric parameters (surface gravity, $\log g$, and effective temperatures, $T_{\text {eff }}$ ) of stars belonging to an SSP with a given age, metallicity, and $[\alpha / \mathrm{Fe}]$ enhancement. A number of stellar evolutionary stages are defined to be included in the synthesis, such that the integrated light spectrum of the SSP can be generated by combining the spectra of individual stars, according to a given IMF. In the present work, we assume a Salpeter IMF (Salpeter 1955), which is very similar with to Kroupa (2001) in the mass range of old stars.

Spectra of individual stars from empirical or synthetic libraries can be used. Given our aim of fitting particular lines by varying their abundances, empirical stellar libraries are not well suited for investigating how changes in the abundances of individual elements affect the spectral indices, since they are restricted to the abundance patterns of stars in the solar neighborhood.

For this reason, we developed the SynSSP package, which computes the spectra of SSPs with variable abundance ratios. The user can compute a synthetic SSP spectrum by providing some key parameters, as follows: 

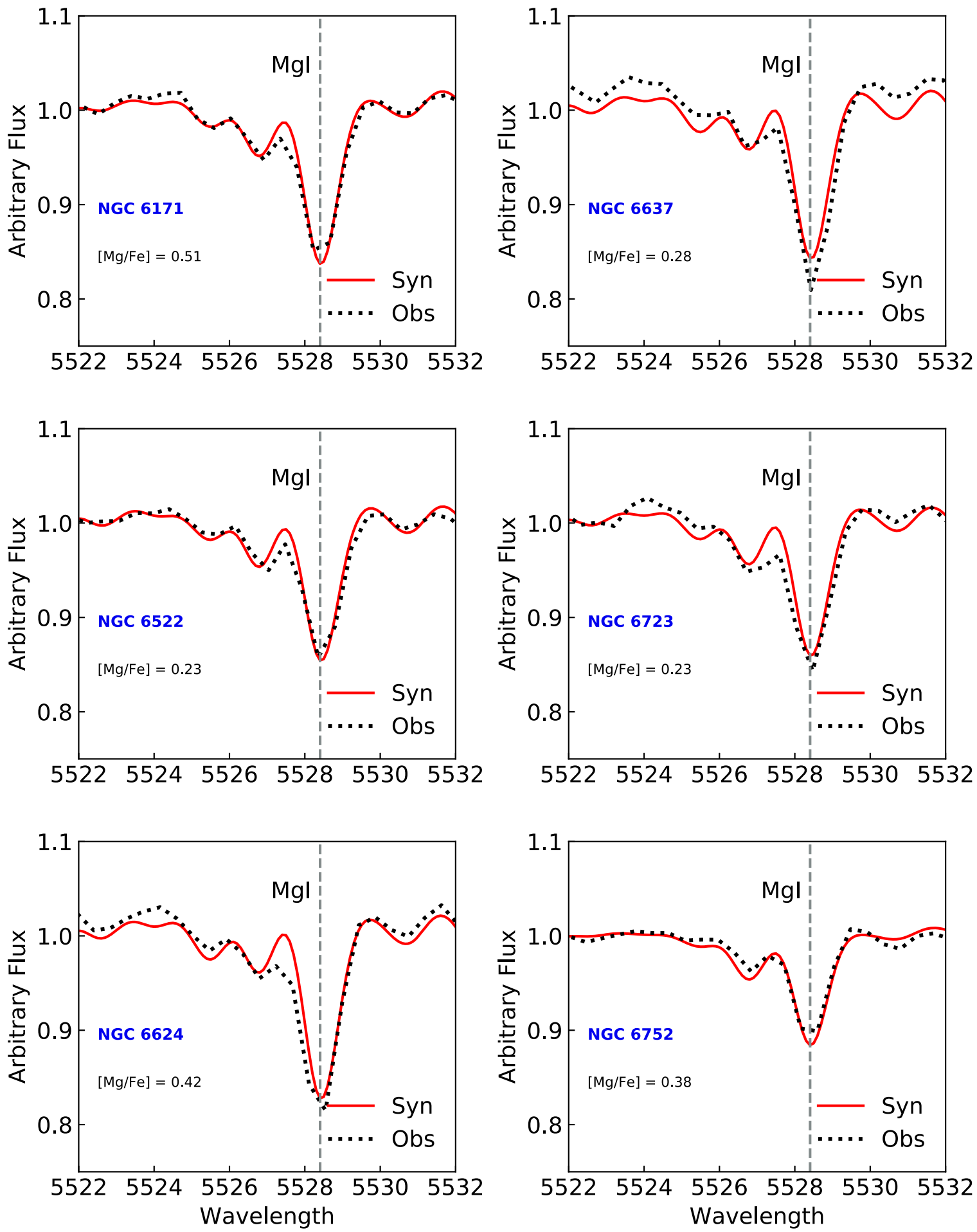

Figure 1. The observed and synthetic spectra for the Mg I line in $5528.405 \AA$ line.

A. Stellar evolutionary ingredients:

1. Age, metallicity, and $[\alpha / \mathrm{Fe}]$ of the SSP. We adopt the Dartmouth $^{4}$ stellar evolutionary tracks (Dotter et al. 2008). Primordial helium abundances are assumed.

2. Number of stars, characterized by their effective temperatures $T_{\text {eff }}$, and surface gravity $\log g$, that should be selected along the isochrone. A standard procedure considers 12

$\overline{4}$ http://stellar.dartmouth.edu/models/index.html stars in the main sequence (MS) and subgiant branch, and 9 stars in the red giant branch (RGB).

B. Synthetic stellar spectra:

1. A stellar synthetic spectrum is computed for each of the $T_{\text {eff }}$ and $\log g$ pairs.

2. The values of $[\mathrm{C}, \mathrm{N}, \mathrm{O}, \mathrm{Na}, \mathrm{Mg}, \mathrm{Al}, \mathrm{Si}, \mathrm{Ca}, \mathrm{Ti}, \mathrm{Ba}$, and $\mathrm{Eu} / \mathrm{Fe}]$ abundance ratios. Some default values are $[\mathrm{C} / \mathrm{Fe}]=$ $-0.2,[\mathrm{~N} / \mathrm{Fe}]=+1.0$, and $[\mathrm{O}, \mathrm{Mg}, \mathrm{Si}, \mathrm{Ca}, \mathrm{Ti}, \mathrm{Eu} / \mathrm{Fe}]=$ $[\alpha / \mathrm{Fe}]$. For the other elements, literature or fitted 

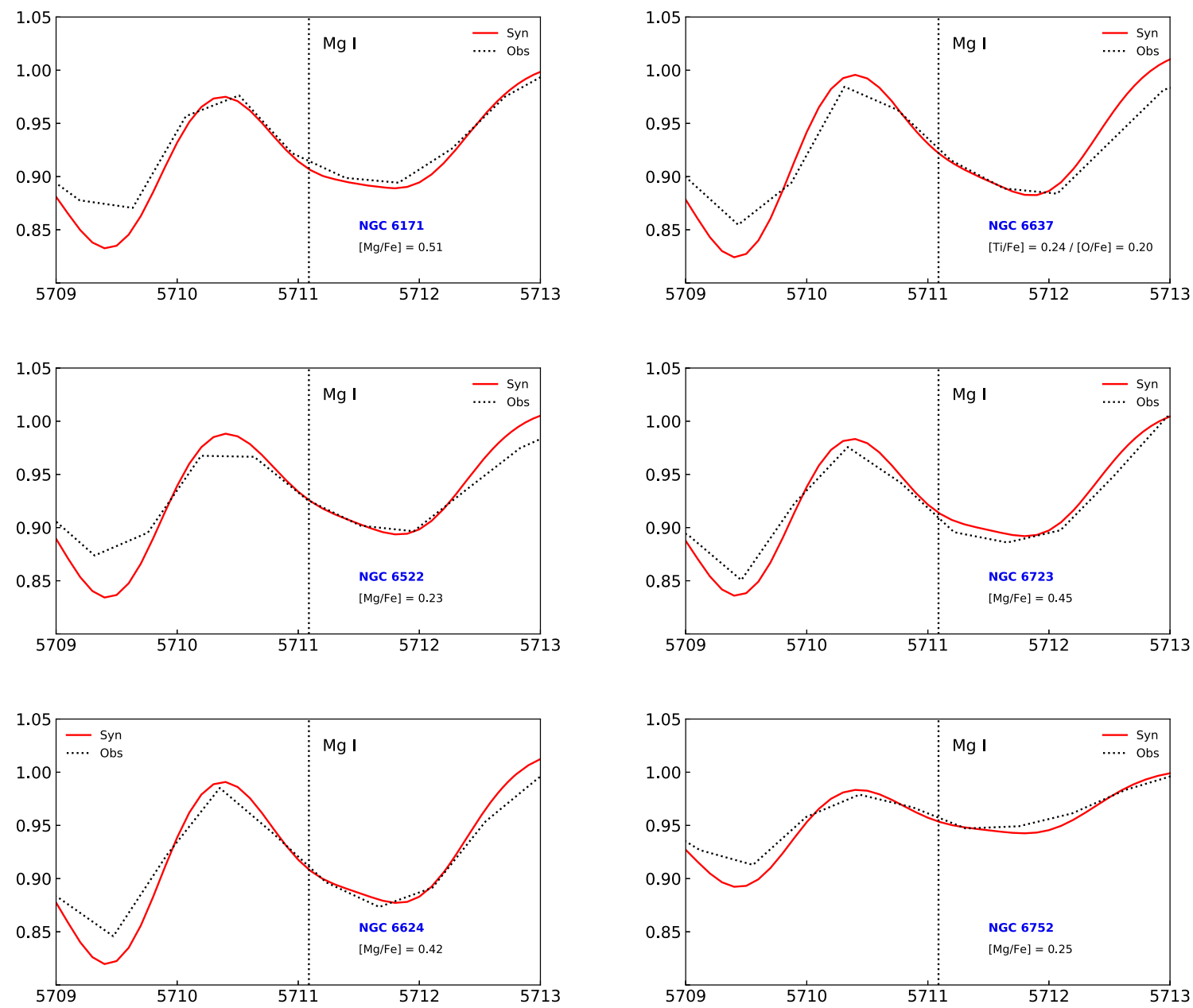

Figure 2. The Mg 5711.088 ̊ line for the sample clusters.

abundances are included, otherwise a solar abundance ratio is assumed.

3. The wavelength range being sampled, the spectral resolution of the computed spectra $\delta \lambda$, and the FWHM for convolution of the synthetic spectra. The default values are $\mathrm{FWHM}=0.2 \AA$ and $\delta \lambda=0.1 \AA$. In order to compare to the sample observed spectra, we used $\mathrm{FWHM}=1.0 \AA$ and $\delta \lambda=0.5 \AA$.

The synthetic stellar spectra are generated using the PFANT code described in Barbuy (1982), Cayrel et al. (1991), Barbuy et al. (2003), and Coelho et al. (2005), and are updated in Barbuy et al. (2018b). Given a stellar model atmosphere and lists of atomic and molecular lines, the code computes a synthetic spectrum assuming local thermodynamic equilibrium (LTE). The atomic line list is that of VALD3 (Ryabchikova et al. 2015). For some specific lines we used updated oscillator strengths, in particular for the Mg I triplet at 5167-5183 $\AA$ (Pehlivan Rhodin et al. 2017). The molecular line list calibrated through several stellar spectroscopic studies is described in Barbuy et al. (2018b). The MARCS LTE atmospheric models by Gustafsson et al. (2008) are employed.

For each star along the isochrone we compute the synthetic spectrum, taking into account the abundances derived from high-resolution spectroscopy initially, and, if needed, further fitting abundances for the line list given in Table 3.

C. Computation of the integrated spectra:

The spectra of the SSP are created using the following integral

$$
f(\lambda)=\int_{m_{1}}^{m_{2}} s(\lambda, m) \phi(m) d m,
$$

where $s(\lambda, m)$ is the spectrum of an individual star with mass $m$ as a function of wavelength $\lambda$ and $\phi(m)$ is the IMF.

The code reads a list of the stellar parameters $T_{\text {eff }}, \log g$, mass, and luminosity $\log \left(L / L_{\odot}\right)$, and the corresponding synthetic stellar spectra $S(\lambda$, mass), computed for each pair of $T_{\text {eff }}, \log g$. The stellar population is then divided into $N$ mass bins, with a lower mass $m_{1}=0.08 M_{\odot}$, and $m_{2}$ is the highest mass found in the isochrones being fitted.

The package that computes SSP spectra with variable chemical abundances is available under request.

The abundances are varied all together, which is important, due in particular to effects of some of them as electron donors. $\mathrm{Fe}, \mathrm{Al}$, and $\alpha$-elements such as $\mathrm{Ca}$, are electron donors, and if enhanced, they contribute to the increase of the continuum by H-. For this reason, it is important to enhance them at the same time. Therefore, our method is different from those by Conroy et al. (2018) or Trager et al. (1998), where each element is 

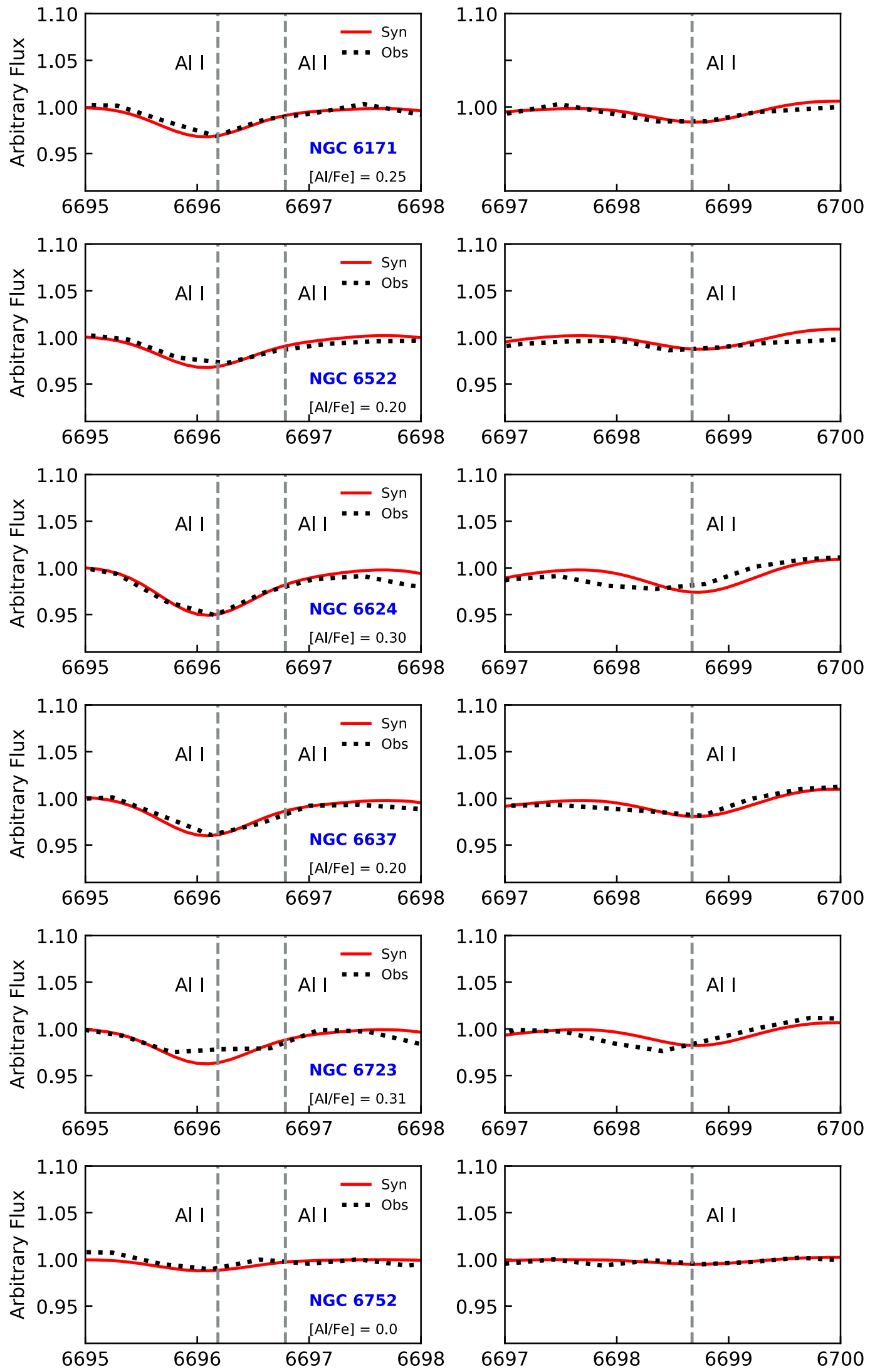

Figure 3. Observed and synthetic spectra in region of the Al I lines.

enhanced separately; it is also different from the method by Colucci et al. (2017) where the integrated spectra are analyzed by measuring EWs of lines.
Our method is more similar to that by Sakari et al. (2013) and Larsen et al. (2017, and references therein), consisting of full spectrum fitting, with the difference of us 

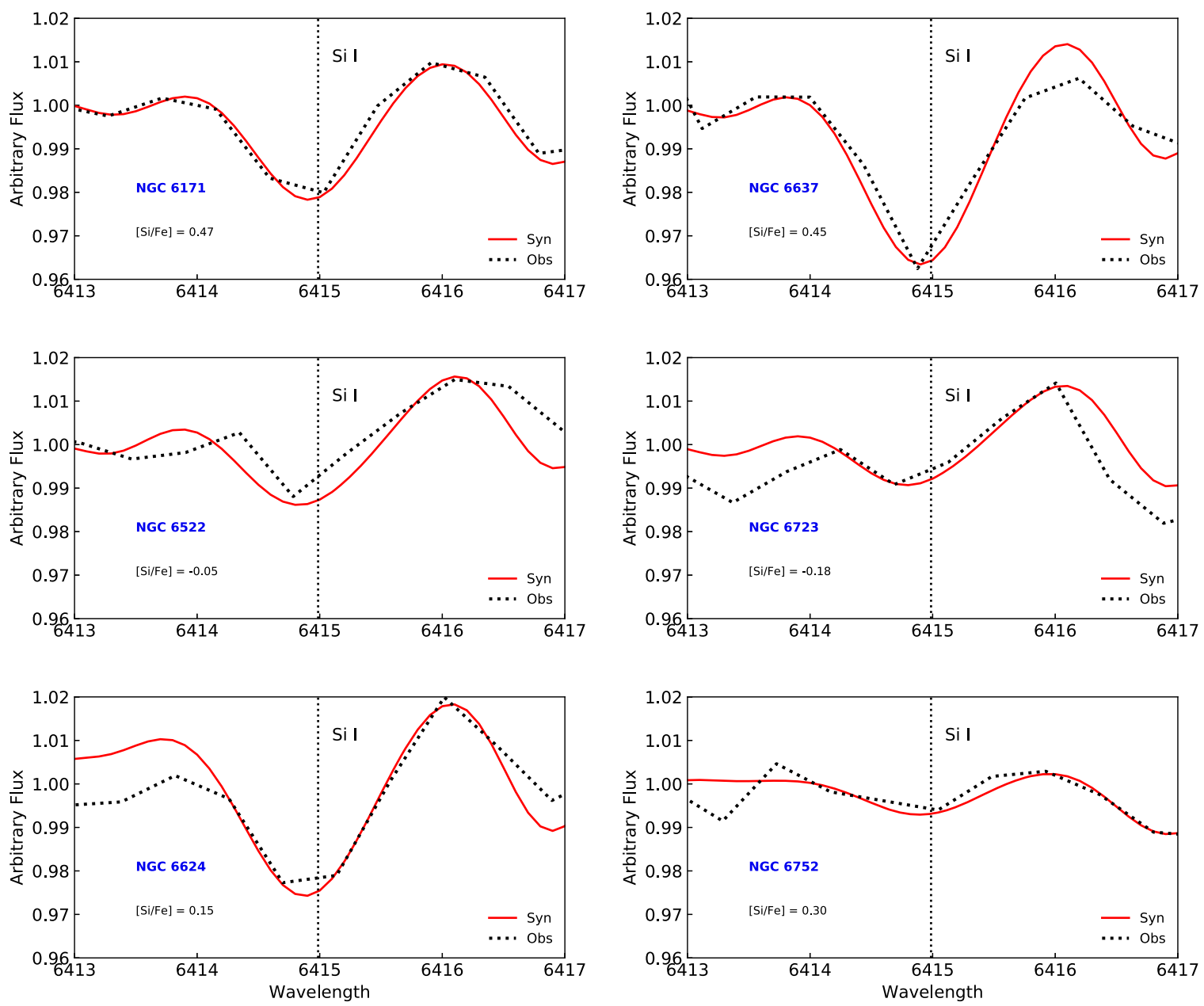

Figure 4. The synthetic and observed spectra for the six clusters of our sample in the region of the $6414.987 \AA$ Si line.

fitting individual selected lines for element abundance derivation.

\section{Reproduction of Integrated Spectra of Sample Clusters}

We have computed integrated spectra for the six sample GCs, using the prescriptions described in the previous section. The overall fit in the wavelength range 4500-9000 $\AA$ is very satisfactory, as can be seen in Figures 15-19 in the Appendix, where where the full spectrum of NGC 6522 is fit. Residuals for regions of $100 \AA$ were measured, and the resulting ratios between the synthetic and observed spectra are below $2 \%$.

We further inspected suitable lines of interesting elements, for which we are able to fit element abundances. The fits to the selected lines, reported in Table 3 , proceeded as in highresolution spectroscopy, i.e., by fitting the local continuum, and finding the best abundance. For the strong lines (Table 4), the abundance is unchanged, because the bottom of these lines cannot be fitted in LTE, as discussed in Section 4.2.

\subsection{Lines of MgI, Na I, AlI, Si I, Ca I, Ti I, Ba II, and Eu II}

In this subsection we present the resulting abundances from spectrum fitting for best lines for the elements $\mathrm{Mg} \mathrm{I}, \mathrm{Na} \mathrm{I}, \mathrm{Al} \mathrm{I}$, Si I, Ca I, Ti I, Ba II, and Eu II. The results are adopted as the element abundance in the sample clusters. The reason we prefer weaker lines rather than stronger lines to derive abundances is the simple fact that in that case the abundance increases linearly with EW (Gray 2005).

The fits to lines for all six clusters, for chosen lines of $\mathrm{Na}, \mathrm{Mg}, \mathrm{Al}, \mathrm{Si}, \mathrm{Ca}, \mathrm{Ti}, \mathrm{Ba}$, and $\mathrm{Eu}$, are described below. The element abundances obtained or adopted are given in the panels and gathered in Table 3. The $\mathrm{Na}$ lines and abundances are treated separately as reported in Section 5.

The uncertainties in the abundances derived in Table 3 are related to the strength of the line, and whether surrounding blends allow a good continuum fit. The uncertainties given in the table for NGC 6171 are adopted for all clusters for the same respective lines.

Mg I Lines-Figures 1 and 2 show the Mg I 5528.405 and $5711.08 \AA$ lines that are well-fitted for the six clusters.

Al Lines-The Al I 6696.185, 6696.788, 6698.673 $\AA$ lines are shown in Figure 3. The calculations are in a good agreement with the integrated spectra, where for the clusters NGC 6723, NGC 6752, and NGC 6522, solar ratios are adopted. For NGC 6171, there are no $\mathrm{Al}$ abundances available in the literature. NGC 6171, NGC 6624, and NGC 6637 show $\mathrm{Al}$ enhancements that are also found in bulge field stars, where it behaves as an $\alpha$-element (see McWilliam 2016; Barbuy et al. 2018a). Note that $\mathrm{Al}$ enhancements can also be an indicator of a second generation of stellar populations in GCs (Renzini et al. 2015; Bastian \& Lardo 2018). 



Figure 5. The 7405.79, 7415.9, and 7423.51 A Si lines in the synthetic and observed spectra for our sample.

Si Lines-Figures 4 and 5 show the fits to the Si I 6414.99 and $7405.79 \AA$ lines.

Ca Lines-Figure 6 shows the fits to the Ca I $6162.167 \AA$, as well as to the nearby $\mathrm{Na}$ I $6160.753 \AA$ line, with the adopted abundances indicated in the figure and in Table 3 . Usher et al. (2019) derived the $\mathrm{Ca}$ abundances for their full sample clusters, based on measurements of EWs with well-defined continua and the wavelength range of $\mathrm{Ca}$ II triplet lines (CaT). The values from Usher et al. (2019) and the present results are compatible (see Section 4.3). It is important to note that in this subsection we derived the $\mathrm{Ca}$ abundance from weak to medium lines, which are more suitable for abundance derivation, therefore a method different from Usher et al. (2019), even if using the same spectra. It is reassuring to conclude that the $\mathrm{Ca}$ abundances from the CaT strong lines measured as indices, and the fit to normally weak to medium lines in the integrated spectra, give very close results.

Ti Lines-Ti I 5965.825, 6261.106, and 6336.113 $\AA$ are used to derive $\mathrm{Ti}$ abundances, with the first two lines shown in Figure 7.
Ba lines-Figure 8 shows the fits to the Ba II $6496.897 \AA$ line, with abundances indicated in the panels and in Table 3. Hyperfine structure (HFS) is taken into account following Barbuy et al. (2014).

Eu Line-Figure 9 gives the Eu II $6645.064 \AA$ line wellfitted with literature abundances when available (NGC 6522 and NGC 6637), and for the others the line was fitted, with the resulting Eu abundance indicated in the figures, and in Table 3. HFS is taken into account.

\subsection{Strong Mg I and Ca II Triplet Lines}

For the strong lines of the Mg I triplet at 5167-5183 $\AA$, and the Ca II triplet at 8498-8662 $\AA$, we have adopted oscillator strengths from the recent literature, and derived astrophysical values for the damping constants to reproduce their wings, applied to the Sun and Arcturus. The abundances are adopted from the lines reported in Table 3.

The main mechanism of collisional broadening for metallic lines in stars of spectral types $\mathrm{F}$ and $\mathrm{M}$ is due to collisions with neutral hydrogen atoms. The damping constant $\gamma$ for each 

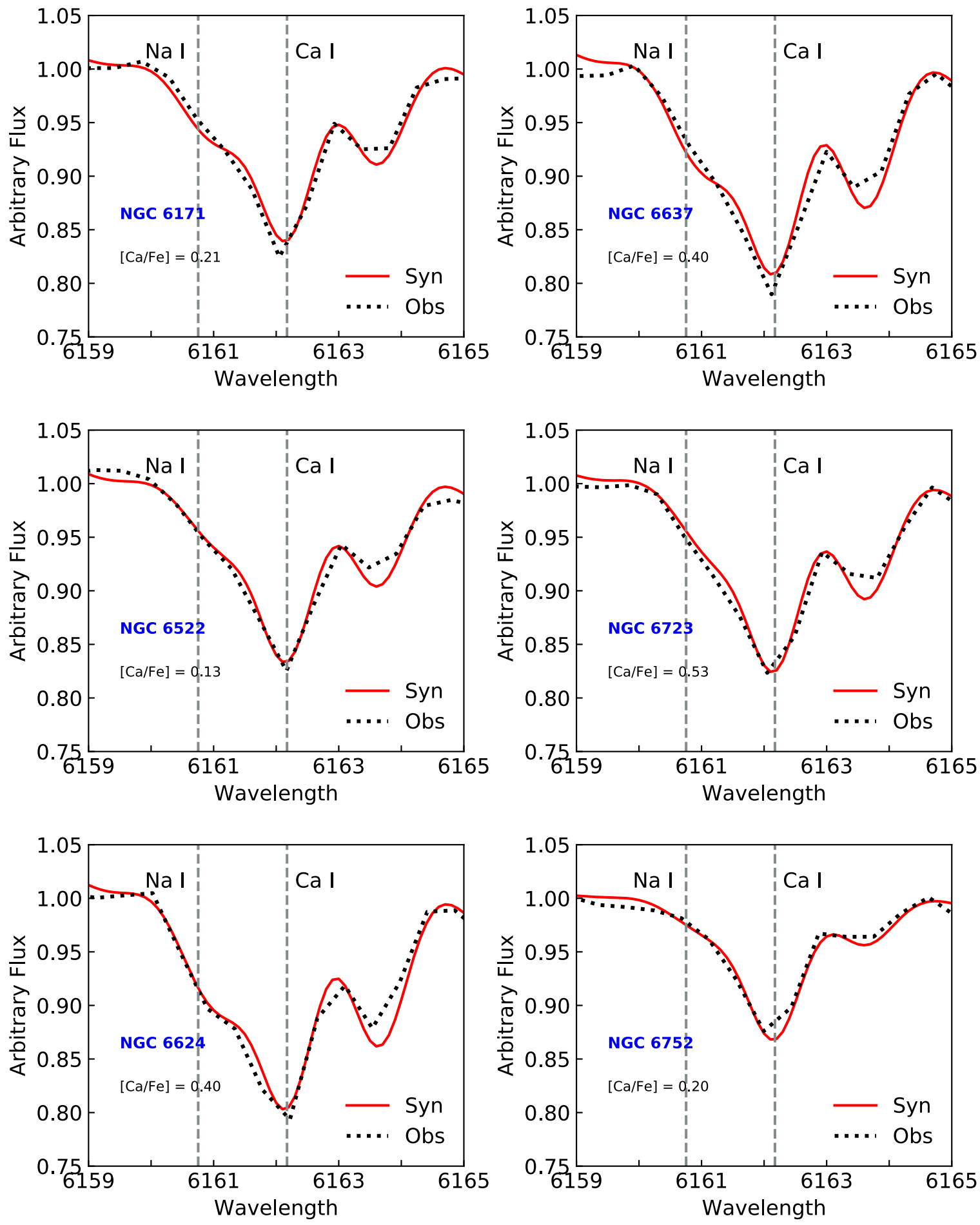

Figure 6. Observed and synthetic spectra in the region of the Ca I $6162.7 \AA$ line.

atmospheric layer relates to the interaction constant $C_{6}$ as follows (Gray 2005):

$$
\gamma_{6} / N_{\mathrm{H}}=17 v^{3 / 5} C_{6}^{2 / 5}
$$

where $v$ is the relative velocity between the colliding particles and $N_{\mathrm{H}}$ is the density of hydrogen atoms.

The radiative broadening (taken as standard for most lines with $\gamma_{\mathrm{R}}=2.21 E 15 / \lambda^{2}$ ), as well as electron broadening are also taken into account, to form the final Voigt profile (Gray 2005).
The values of $\gamma_{\mathrm{R}}$ and $\gamma_{\mathrm{e}}$ are adopted from the VALD3 (Ryabchikova et al. 2015) and Kurúcz webpage, ${ }^{5}$ and these are further described in Smith \& Drake (1988) and Chmielewski (2000).

The adopted values of the interaction constant, $C_{6}$, were obtained by adopting the recent literature oscillator strengths, and fitting of the wings. This is done by using the MARCS

5 http://www.cfa.harvard.edu/amp/ampdata/kurucz23/sekur.html 

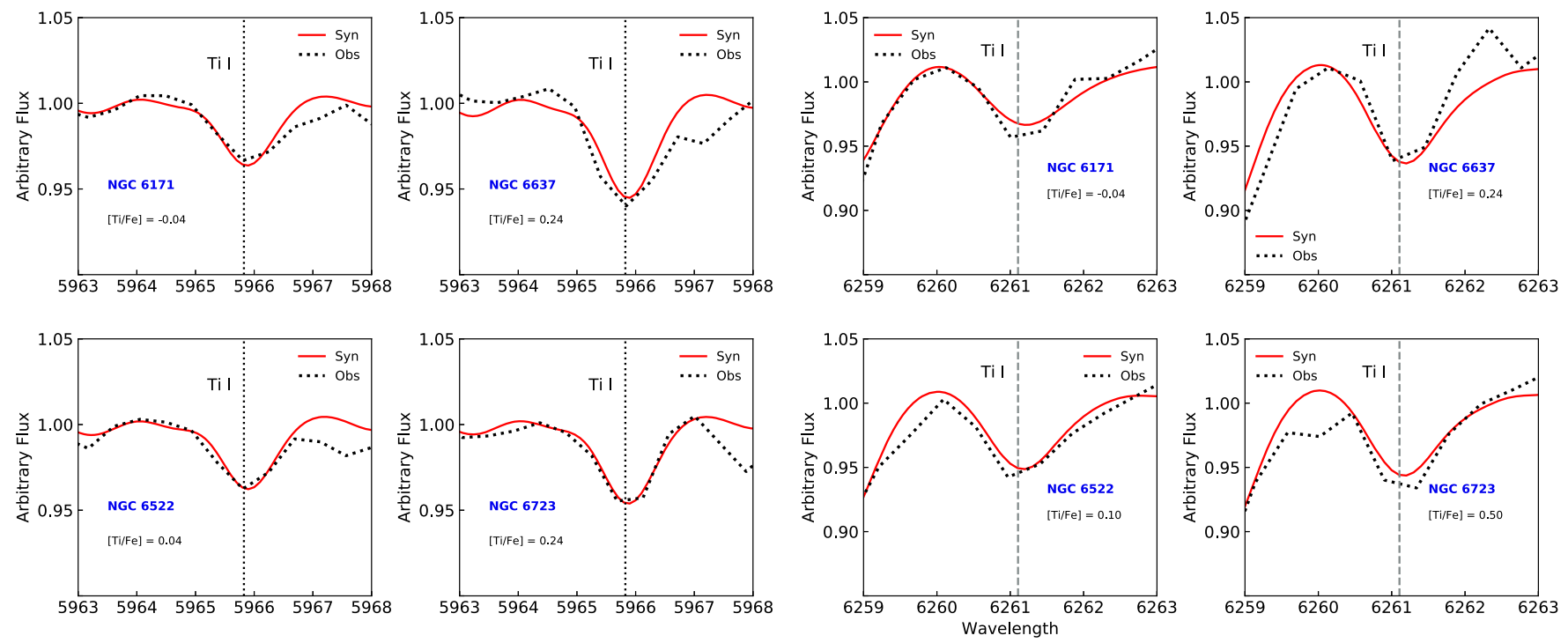


(a)
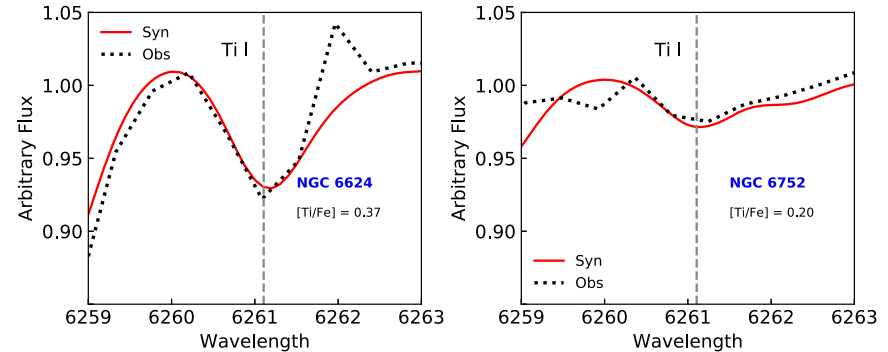

(b)

Figure 7. Fitting of synthetic to observed spectra for different regions with Ti I lines.

models (Gustafsson et al. 2008) applied to the Sun (Kurúcz $2005)^{6}$ and Arcturus (Hinkle et al. 2000). We adopted the stellar parameters $T_{\text {eff }}, \log g$, metallicity $([\mathrm{Fe} / \mathrm{H}])$, and microturbulent velocity $\left(v_{t}\right)$ of $5770 \mathrm{~K}, 4.44,0.0$, and $1.0 \mathrm{~km} \mathrm{~s}^{-1}$ for the Sun, and $4275 \mathrm{~K}, 1.55,-0.54,1.65 \mathrm{~km} \mathrm{~s}^{-1}$ for Arcturus, from Meléndez et al. (2003). We adopted abundances of $A(\mathrm{Fe})=7.50$ for the Sun, and $[\mathrm{Mg} / \mathrm{Fe}]=+0.15$ and $[\mathrm{Ca} / \mathrm{Fe}]=+0.10$ for Arcturus. Note that the damping constant through the interaction constant $C_{6}$ that fit the wings of strong lines, depends on the atmospheric model grid adopted, as pointed out in Barbuy et al. (2003, their Figure 1).

Another comment regarding strong lines is that LTE calculations do not reproduce the bottom of these lines, given that they form in NLTE, and besides their formation takes place in chromospheric layers, that are not included in the usual model atmospheres.

For the Mg I triplet lines at 5167.3, 5172.68, and 5183.60 and $\mathrm{CaT}$ at 8498.023, 8542.091, and 8662.141 $\mathrm{A}$, we used the atomic constants listed in Table 4 . This resulted in very good fits shown in Figures 10 and 11.

\subsection{Literature Abundances}

The resulting abundances derived in the above subsections are then compared with other methods of abundance derivation from integrated light reported in the literature. The element abundances derived from different methods and sources are compiled in Table 2. The method employed is identified as high-resolution

\footnotetext{
6 kurucz.harvard.edu/Sun.html
}

spectroscopy (HRS), CMD, compilation (comp.), and from integrated spectra: equivalent widths (ISEW), full spectrum fitting (ISFF), or finally the fit of individual lines (ISfit), the latter being our adopted method.

Below we briefly describe methods employed in the literature for derivation of abundances applied to the six sample GCs. The following references and comments given here are by no means exhaustive, since there is more extensive literature addressing the clusters.

Usher et al. (2019) derived $\mathrm{Ca}$ abundances from EW measurements following prescriptions given in Usher et al. (2012), similarly to other studies in the literature such as Vásquez et al. (2018) applied to individual stars, or Foster et al. (2010) applied to integrated light. Usher et al. (2019) derive Ca abundances for all sample clusters in the present work and their values are given in Table 2 .

Colucci et al. (2017), following earlier work by Sakari et al. (2013), measured EWs of individual lines of $\mathrm{Mg}, \mathrm{Ca}, \mathrm{Ti}, \mathrm{Cr}$, $\mathrm{Ba}$, and $\mathrm{Eu}$, from the same WAGGS spectra used here, and they report the corresponding element abundances. They also use spectrum synthesis to fit iron peak and heavy element lines that require inclusion of HFS. The cluster NGC 6752 is a common factor in Colucci et al. (2017) and the present work, noting that in most cases Colucci et al. chose different lines relative to our Table 3. In Table 5, we compare the abundances for the lines in common between Colucci et al. and the present work, for NGC 6752.

Larsen et al. (2017) obtained high-resolution integrated spectra $(R \sim 40,000)$ of seven clusters, including NGC 6752, in common with the present work. Their method of full 

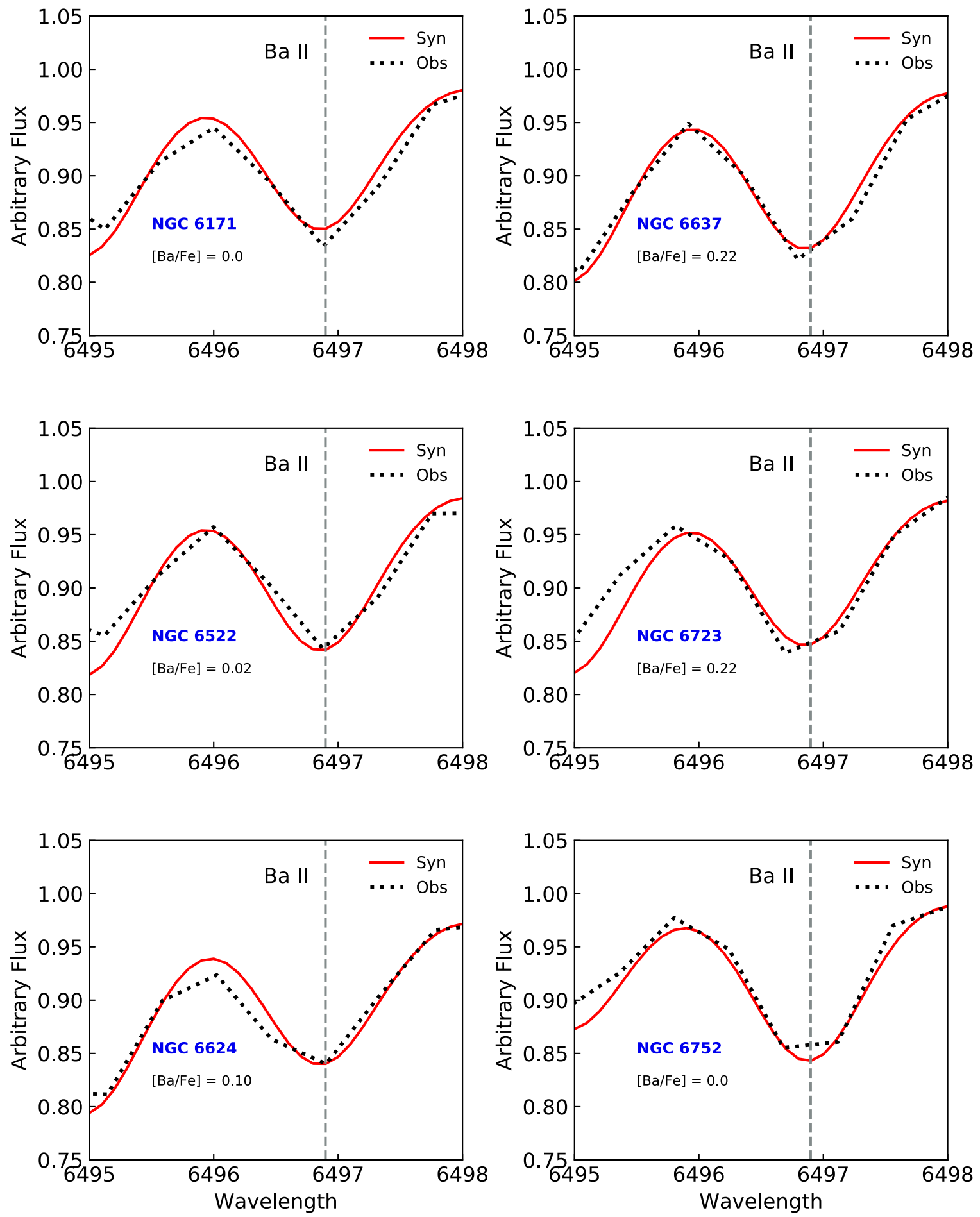

Figure 8. Observed and synthetic spectra in the region of the line $6496 \AA$ Ba II.

spectrum synthesis, as well as the use of the Dartmouth isochrones is similar to that of the present work. They apply overall fitting to all the spectrum, finding the best abundances to fit it all, therefore in this respect, it is different from the present work where we have chosen the most reliable lines to be fitted.

Conroy et al. (2018) present a full spectrum fitting method to derive abundances from integrated spectra. They use Dartmouth isochrones and MILES empirical spectra (SánchezBlázquez et al. 2006), complemented by a library of spectra in the near-infrared. An important addition is the calculation of synthetic spectra with variable abundances, in order to adapt the observed spectra to response functions due to variable abundances. From fitting spectra by Schiavon et al. (2005), they derive abundances of $\mathrm{Mg}, \mathrm{Si}, \mathrm{Ca}$, and $\mathrm{Ti}$ for $41 \mathrm{GCs}$, including our six sample clusters.

Table 6 reports the final mean abundance values obtained in the present work. In Figure 12 these results are compared with those from the literature listed in Table 2, for the elements $\mathrm{Mg}$, $\mathrm{Al}, \mathrm{Si}, \mathrm{Ca}$, and $\mathrm{Ti}$. We do not include $\mathrm{Na}$, for the reasons 

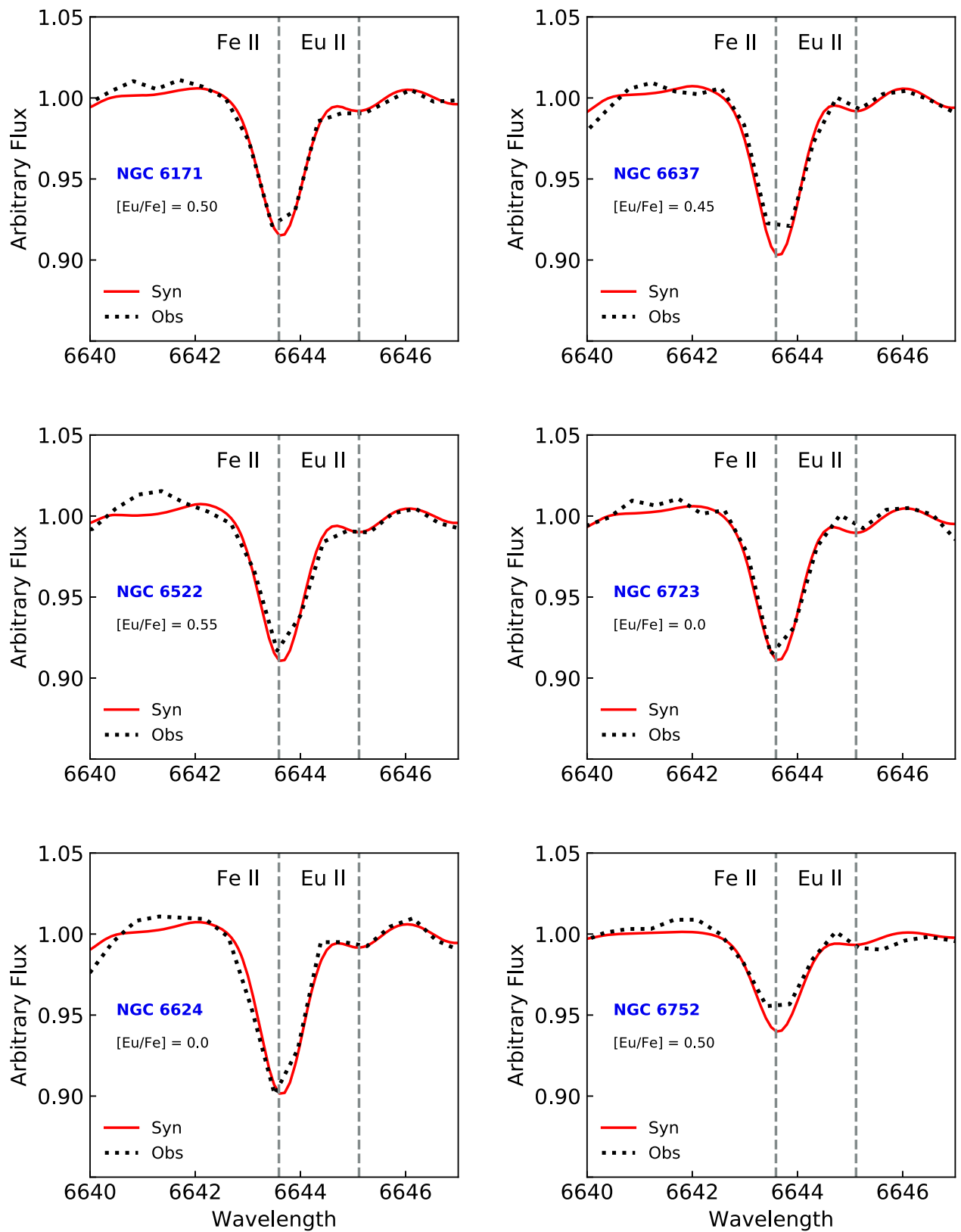

Figure 9. The $6645 \AA$ Eu II line in the synthetic and observed spectra for each globular cluster of our sample.

explained in Section 5 (i.e., the difficulty in deriving reliable $\mathrm{Na}$ abundances from the lines available), or $\mathrm{Ba}$ and Eu because there are very few literature abundances for these elements for the sample clusters. Figure 12 shows that there is good agreement between the present results and other abundance derivations from integrated spectra-star symbols (ours) compared with squares (others). A spread of abundances is seen among different high-resolution spectroscopic analyses: for NGC 6522, the present results are in good agreement with our previous high-resolution spectroscopic analysis (Barbuy et al. 2014), and there are differences with respect to Ness et al. (2014). For NGC 6752, the high-resolution spectroscopic analysis by Carretta et al. (2012) gives abundances for its three stellar populations and a mean value is plotted in the figure. S. O. Souza et al. (2019, in preparation) found fractions of $25 \%$, $43 \%$, and $32 \%$ for $1 \mathrm{G}, 2 \mathrm{G}$, and $3 \mathrm{G}$, very similar to the proportions of $25 \%, 40 \%$, and $30 \%$ obtained by Milone et al. (2013), and also adopted by Tenorio-Tagle et al. (2019), therefore a mean of abundances from the three stellar populations appears suitable. Aluminum has a high abundance, given their high abundance values for $2 \mathrm{G}$ and $3 \mathrm{G}$ stars, that we do not confirm from the integrated spectra.

In conclusion, the derivation of abundances line-by-line from integrated spectra, for a few suitable lines, does give results compatible with high-resolution spectroscopic analyses, and with other integrated spectra analyses.

\section{Effects of Multiple Stellar Populations}

The identification of multiple stellar populations in GCs has been recently definitively demonstrated by the so-called chromosome map method, with UV photometric data provided in Piotto et al. (2015), and explained in detail in Milone et al. (2017). For many of the present clusters, the identification of percentages of first and second stellar populations are available 

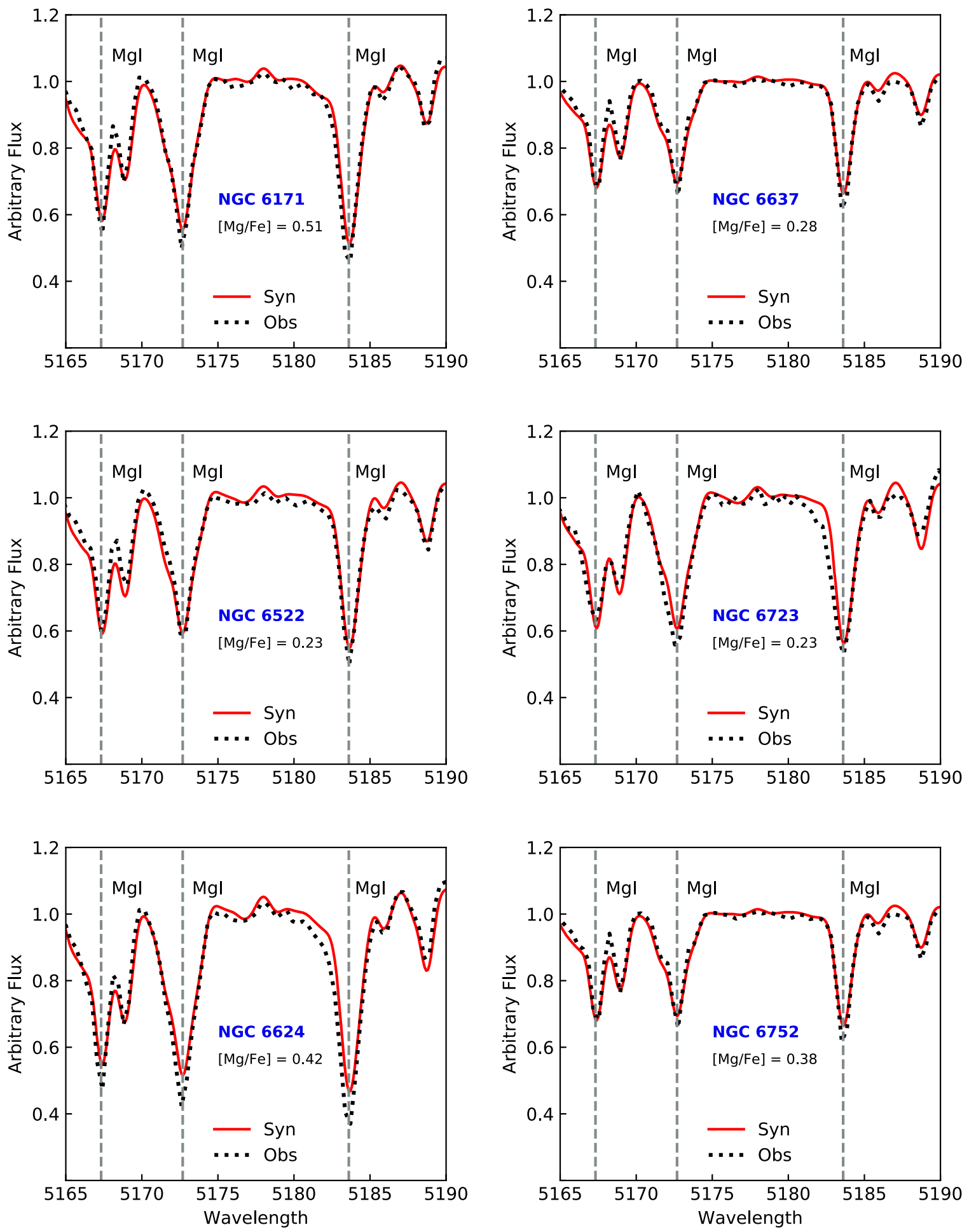

Figure 10. The Mg I triplet lines at 5167.3, 5172.68, and 5183.60 A for the sample clusters.

in Milone et al. (2017). The reason for the chromosome map to be feasible is due to the variations in the abundances of $\mathrm{C}, \mathrm{N}$, and O, as clearly illustrated in Figure 1 by Piotto et al. (2015).

The presence of multiple stellar populations together with variations in abundances of $\mathrm{C}, \mathrm{N}, \mathrm{O}, \mathrm{Na}, \mathrm{Al}$, and $\mathrm{Mg}$ was identified and described in Carretta et al. (2009), Gratton et al. (2012), Renzini et al. (2015), and Bastian \& Lardo (2018), among others. Anticorrelations $\mathrm{Na}-\mathrm{O}$ and $\mathrm{Mg}-\mathrm{Al}$ are observed, where in particular the $\mathrm{Na}-\mathrm{O}$ behavior has been extensively documented (Carretta 2019; Carretta et al. 2009). Note that for $\mathrm{C}, \mathrm{N}$, and $\mathrm{O}$, other effects such as stellar evolution along the RGB also affect their abundances.

Na overabundances - The main effect in abundances of second generation stars is the $\mathrm{Na}-\mathrm{O}$ anticorrelation as shown in Carretta et al. (2009) and Carretta (2019) for a sample of GCs. In second generation stars, $\mathrm{Na}$ is enhanced by amounts between 0.2 and 0.7 

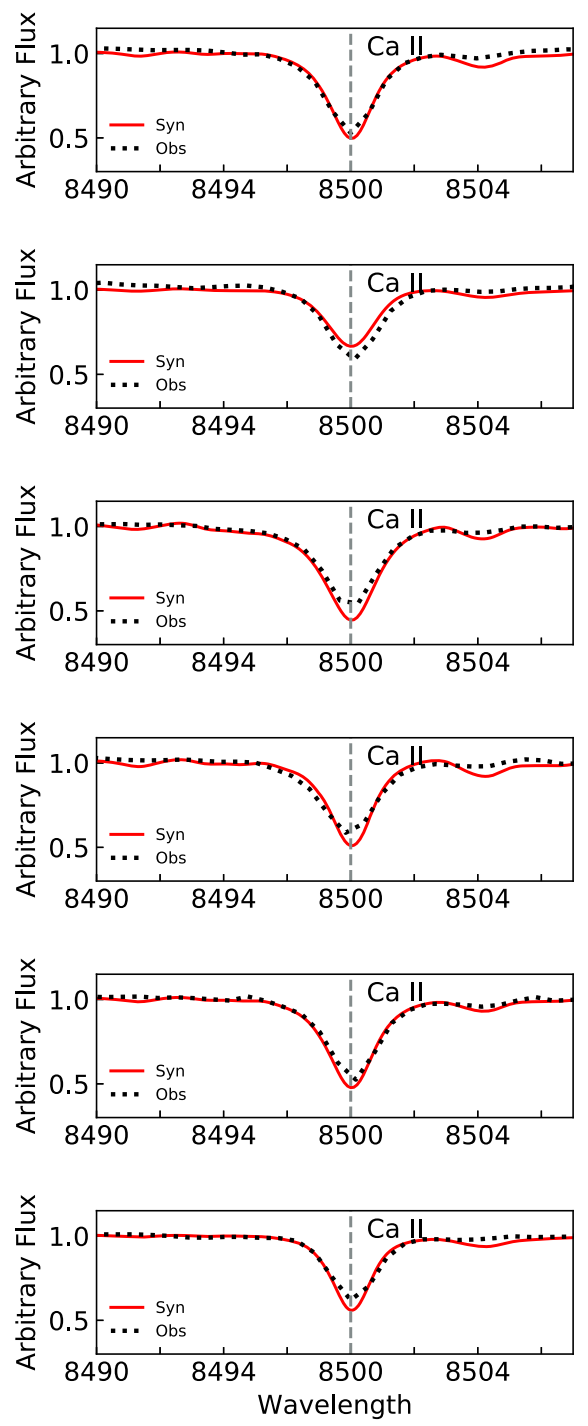
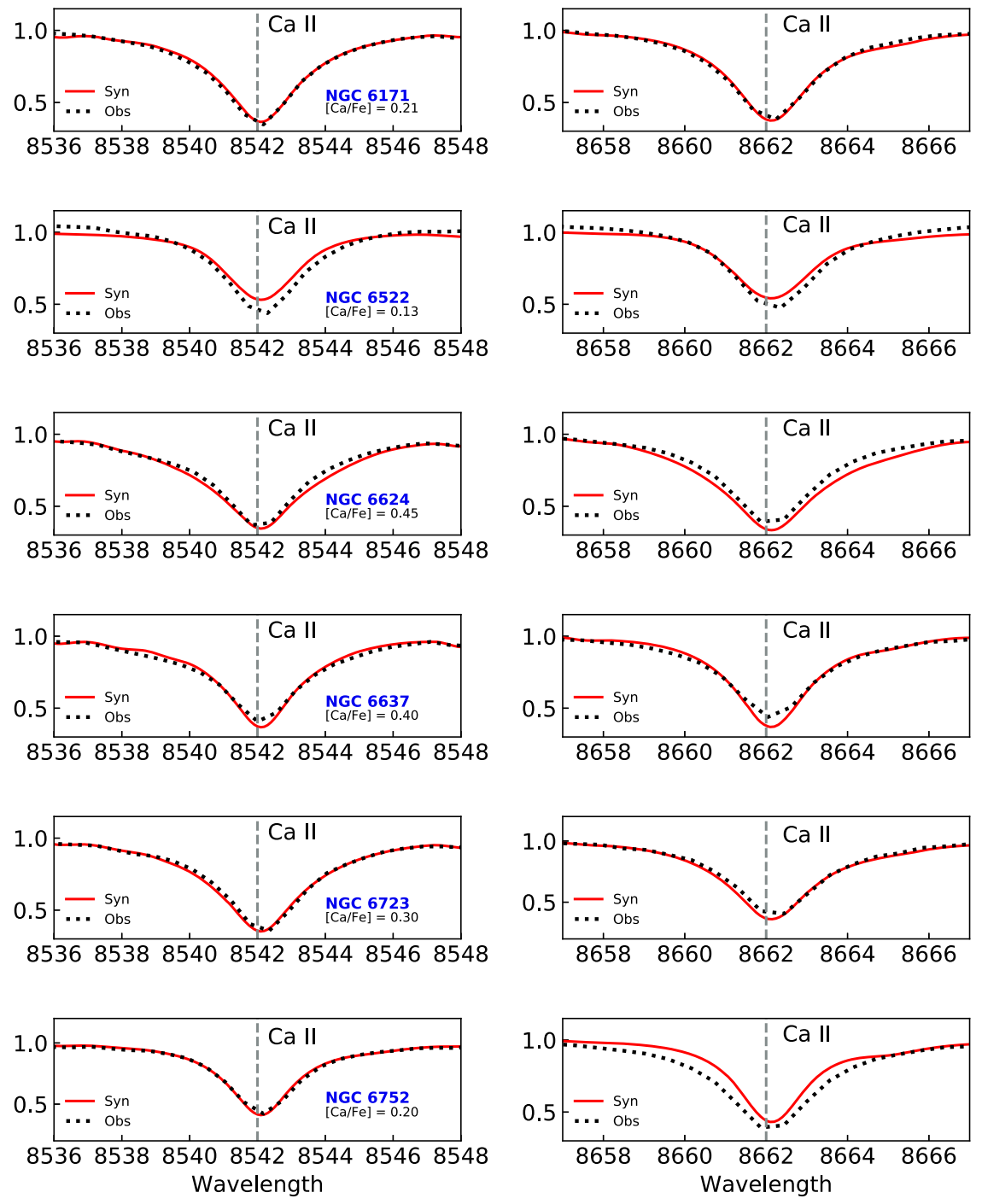

Figure 11. Ca II triplet lines for the sample clusters.

(Campbell et al. 2013), whereas oxygen is depleted. The proportions of first generation stars relative to total $N_{1} / N_{\text {total }}$ were measured by Milone et al. (2017), and confirmed in R. A. P. Oliveira et al. (2019, in preparation), in a first approximation, as given in Table 1. If only bonafide members of first and second generations are taken into account, the proportions can change in some cases (R. A. P. Oliveira et al. 2019, in preparation). For the case of NGC 6522, according to Kerber et al. (2018), 84\% of the stellar population belongs to a second generation, therefore its first generation fraction should be around $N_{1} / N_{\text {total }}=0.160$. In most cases, the second generation is dominant, and this should be seen in the integrated spectra.

Figure 13 shows the fit of three doublets of $\mathrm{Na} \mathrm{I}$, assuming a mean $[\mathrm{Na} / \mathrm{Fe}]=0.0$ and +0.35 , for lines in NGC 6723. These values are chosen from Table 2 where the highest mean values of $[\mathrm{Na} / \mathrm{Fe}]=0.35$ are given for some of the sample clusters. The graph for NGC 6723, exemplifies the difficulty in establishing which is the mean Na abundance. The line NaI $5682.63 \AA$ is inconclusive, line $8194.79 \AA$ indicates $[\mathrm{Na} / \mathrm{Fe}]=0.0$, whereas $5688.20,6154.23$, and $6160.75 \AA$ indicate that $[\mathrm{Na} / \mathrm{Fe}]=+0.35$, and $8183.26 \AA$ is in between. In Table 7 we report the indication of mean $\mathrm{Na}$ abundance from this set of lines for the sample clusters. The conclusion is that the present data do not allow us to draw firm evidence of second generation stars signatures, and that higher $\mathrm{S} / \mathrm{N}$ and higher resolution data would probably allow such a procedure.

Other elements that could be indicators of multiple stellar populations, $\mathrm{Mg}$ and $\mathrm{Al}$, are normally enhanced as found in bulge stars (McWilliam 2016; Barbuy et al. 2018a). It would be possible to infer multiple stellar population evidence for a cluster or galaxy if $\mathrm{Na}$ is very high and/or $\mathrm{Mg}$ is very low.

IMF and enhancements of $\mathrm{Na}$ and $\mathrm{TiO}$ features-Note that $\mathrm{Na}$ enhancements are also used to derive evidence on bottomheavy IMFs (Conroy \& van Dokkum 2012). As concerns Na, given that in our Galactic bulge there is no evidence of IMF variations (Wegg et al. 2017; Barbuy et al. 2018a), we assume standard IMF throughout this paper. We believe that $\mathrm{Na}$ lines are probably not suited for IMF deduction, given the effects not only of multiple stellar populations discussed in the previous subsection, but also because $\mathrm{Na}$ can be enhanced in the central parts of galaxies, as it apparently is in our Galactic bulge (Lecureur et al. 2007; Barbuy et al. 2018a). 


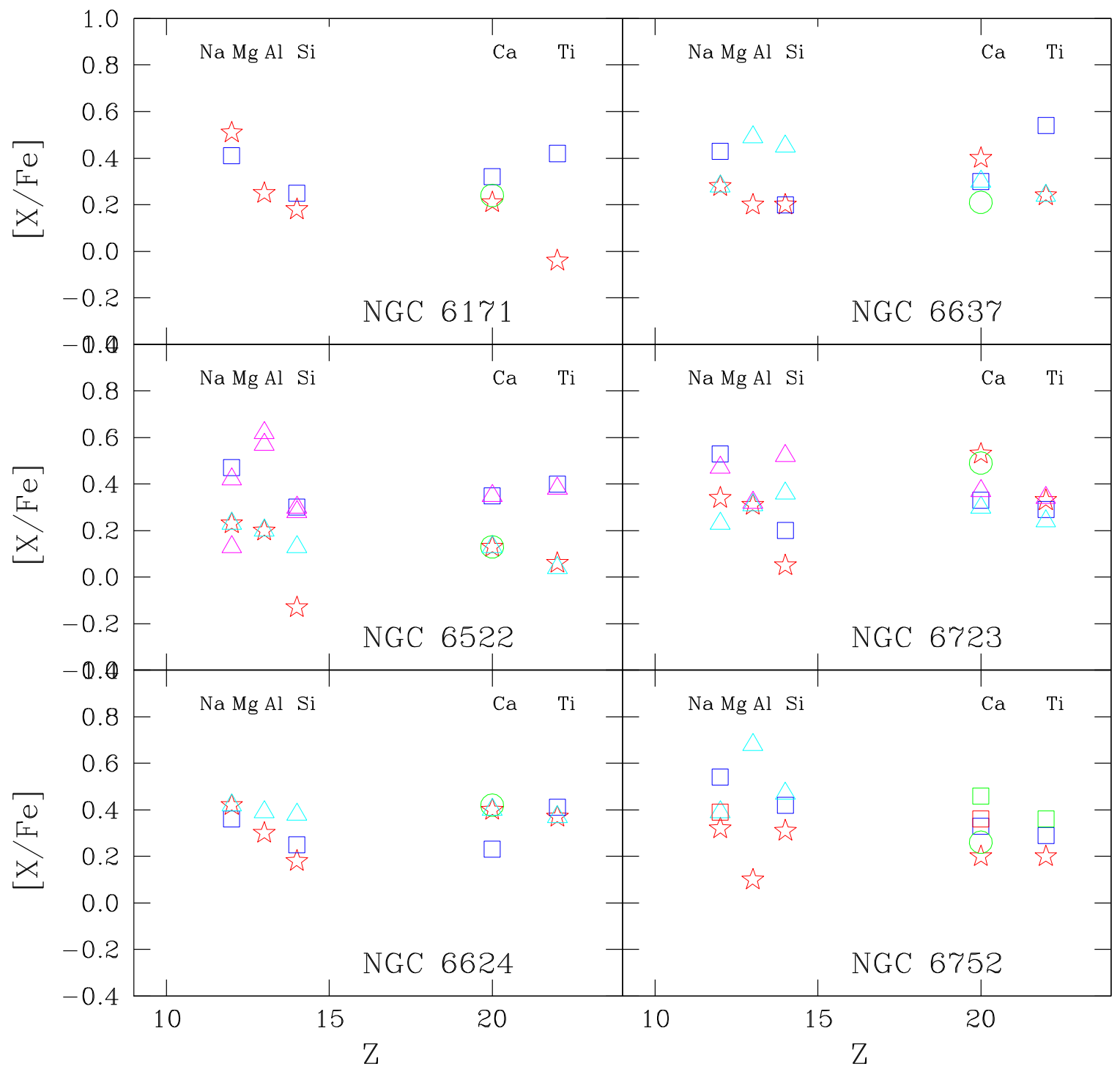

Figure 12. $[\mathrm{X} / \mathrm{Fe}]$ vs. element number $Z$. Symbols given in all panels: present results: red open stars; Conroy et al. (2018): blue open squares; Usher et al. (2019): green open circles. Other symbols: cyan open triangles: Barbuy et al. (2014), Valenti et al. (2011), Lee (2007), Rojas-Arriagada et al. (2016), and Carretta et al. (2012); magenta open triangles: Ness et al. (2014) and Crestani et al. (2019); green open squares: Colucci et al. (2017); red open squares: Larsen et al. (2017).

Table 5

Comparison of Abundances Line-by-line with Colucci et al. (2017) for NGC 6752, Assuming Their $[\mathrm{Fe} / \mathrm{H}]=-1.58$

\begin{tabular}{|c|c|c|c|c|c|}
\hline Species & $\lambda(\AA)$ & $A(\mathrm{X})_{\odot \text { Colucci }}$ & $A(\mathrm{X})_{\text {Colucci }}$ & {$[\mathrm{X} / \mathrm{Fe}]_{\text {Colucci }}$} & $\overline{[\mathrm{X} / \mathrm{Fe}]_{\text {thiswork }}}$ \\
\hline $\mathrm{Na} I$ & 5682.633 & 6.44 & 4.95 & +0.09 & +0.00 \\
\hline $\mathrm{Na} I$ & 5688.200 & 6.34 & 4.75 & -0.01 & +0.35 \\
\hline Mg I & 5528.405 & 7.52 & 6.05 & +0.11 & +0.38 \\
\hline $\mathrm{Mg}$ I & 5711.088 & 7.58 & 5.95 & -0.05 & +0.25 \\
\hline Si I & 7405.79 & 7.56 & 6.56 & +0.58 & +0.47 \\
\hline Si I & 7415.96 & 7.63 & 6.46 & +0.41 & +0.47 \\
\hline Si I & 7423.51 & 7.60 & 6.26 & +0.24 & +0.00 \\
\hline $\mathrm{Ca} I$ & 6162.167 & 6.40 & 5.06 & +0.24 & +0.20 \\
\hline $\mathrm{Ca} \mathrm{I}$ & 6166.440 & 6.36 & 5.16 & +0.38 & +0.20 \\
\hline $\mathrm{Ca} \mathrm{I}$ & 6439.080 & 6.02 & 4.96 & +0.52 & +0.20 \\
\hline Ti I & 5866.449 & 5.04 & 4.00 & +0.54 & +0.20 \\
\hline Ba II & 6496.90 & 2.18 & 0.73 & +0.13 & 0.00 \\
\hline
\end{tabular}

Studies on IMF variations are deduced, besides studies using $\mathrm{Na}$ lines, also from $\mathrm{TiO}$ and Wing-Ford $\mathrm{FeH}$ bands (La Barbera et al. 2016). The $\mathrm{FeH}$ bands are located around
$1 \mu \mathrm{m}$ (Schiavon et al. 1997), therefore out of the range of the present data. TiO bands can be investigated with the present method. We tried to compute $\mathrm{TiO}$ bands at $7000-7500 \AA$, as 

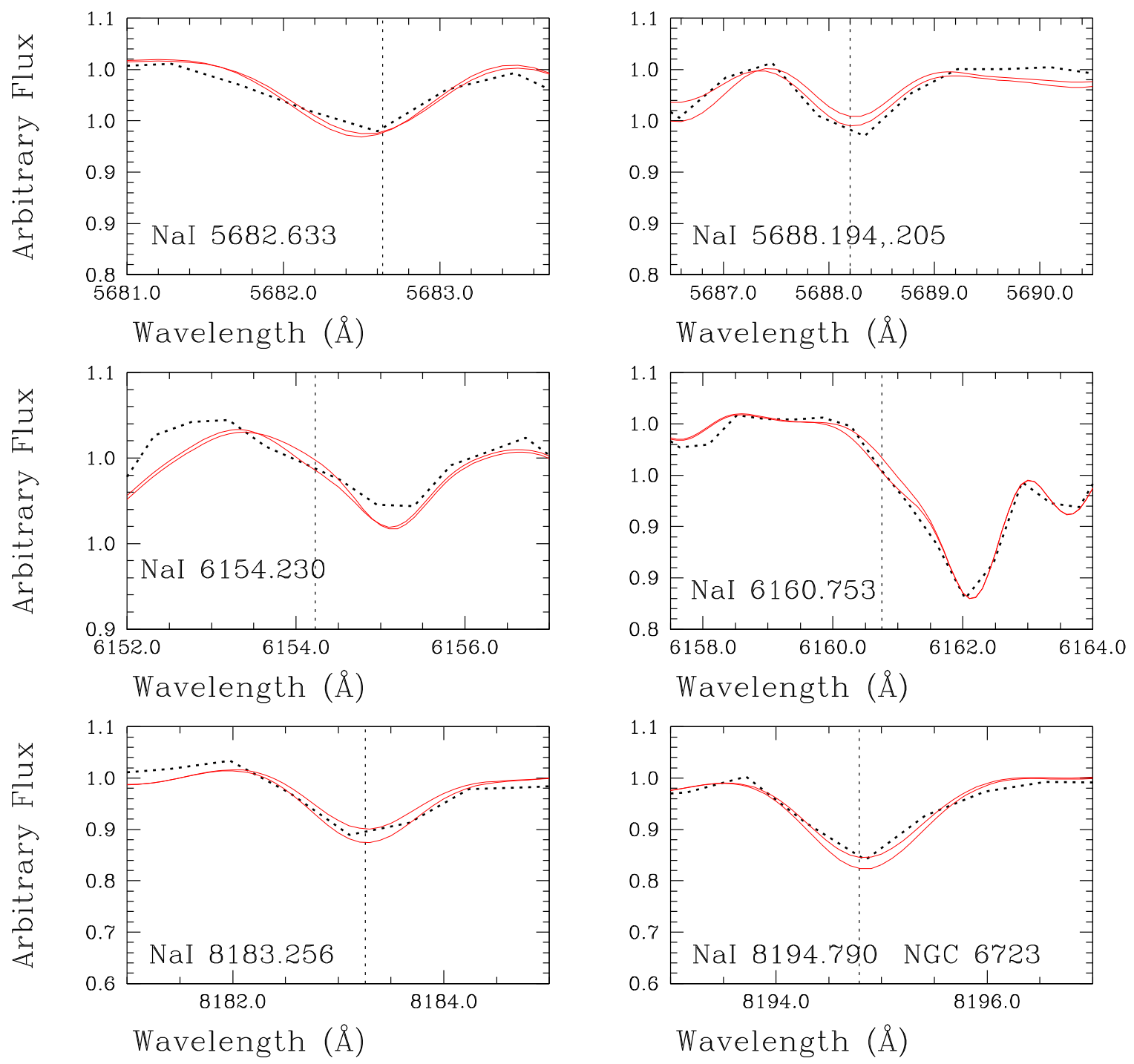

Wavelength $(\AA)$

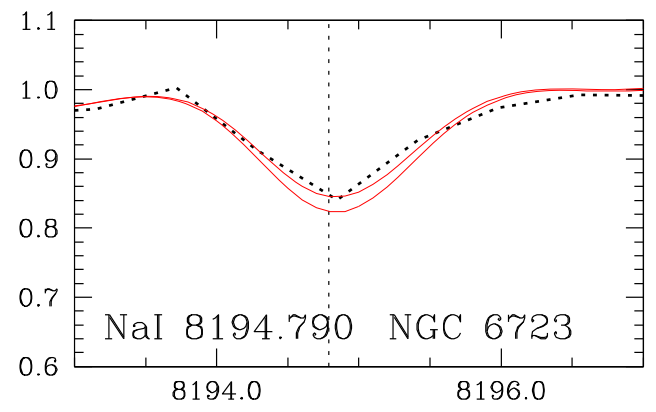

Wavelength $(\AA)$

Figure 13. The observed and synthetic spectra for NGC 6723 of Na I lines: (a) $5682 \AA$ and $5688 \AA$; (b) 6154 and $6160 \AA$; and (c) 8183 and $8194 \AA$, computed with $[\mathrm{Na} / \mathrm{Fe}]=0.0$ and 0.35 .

Table 6

Adopted Metallicities and Resulting Mean Abundances for the Elements: $\mathrm{Mg}, \mathrm{Al}, \mathrm{Si}, \mathrm{Ca}, \mathrm{Ti}, \mathrm{Ba}$, and Eu. Na is given in Table 7

\begin{tabular}{lcccccccc}
\hline \hline Cluster & {$[\mathrm{Fe} / \mathrm{H}]$} & {$[\mathrm{Mg} / \mathrm{Fe}]$} & {$[\mathrm{Al} / \mathrm{Fe}]$} & {$[\mathrm{Si} / \mathrm{Fe}]$} & {$[\mathrm{Ca} / \mathrm{Fe}]$} & {$[\mathrm{Ti} / \mathrm{Fe}]$} & {$[\mathrm{Ba} / \mathrm{Fe}]$} & {$[\mathrm{Eu} / \mathrm{Fe}]$} \\
\hline 6171 & -1.02 & 0.51 & 0.25 & 0.18 & 0.21 & -0.04 & 0.00 \\
6522 & -0.95 & 0.23 & 0.20 & -013 & 0.13 & 0.06 & 0.02 \\
6624 & -0.69 & 0.42 & 0.30 & 0.18 & 0.40 & 0.37 & 0.10 \\
6637 & -0.77 & 0.28 & 0.20 & 0.20 & 0.40 & 0.24 & 0.22 \\
6723 & -0.98 & 0.34 & 0.31 & 0.05 & 0.53 & 0.33 & 0.00 \\
6752 & -1.53 & 0.32 & 0.10 & 0.31 & 0.20 & 0.20 & 0.45 \\
\end{tabular}

previously exemplified in Milone et al. (1995), applied to the most metal-rich cluster in our sample, NGC 6624. However, at this metallicity the bands are not strong enough to be inspected.

Helium abundances-We have adopted isochrones with primordial helium abundances, that in the DSED isochrones correspond to $Y=0.247$. Despite detection of a small helium enchancement in second generation stars for these clusters by Lagioia et al. (2018) and Milone et al. (2018, 2019), the effects on the CMDs, for our purposes, are negligible. The He enhancement in $2 \mathrm{G}$ stars of the sample clusters, from Milone et al. (2019), are reported in Table 1. The enhancements are small, therefore the isochrones for $2 \mathrm{G}$, if He enhancement was to be taken into account, would be very close to the $1 \mathrm{G}$ enhancement. Essentially, if enhanced He is assumed, there is a small decrease in age. S. O. Souza et al. (2019, in preparation) have studied the three stellar populations in NGC 6752, 

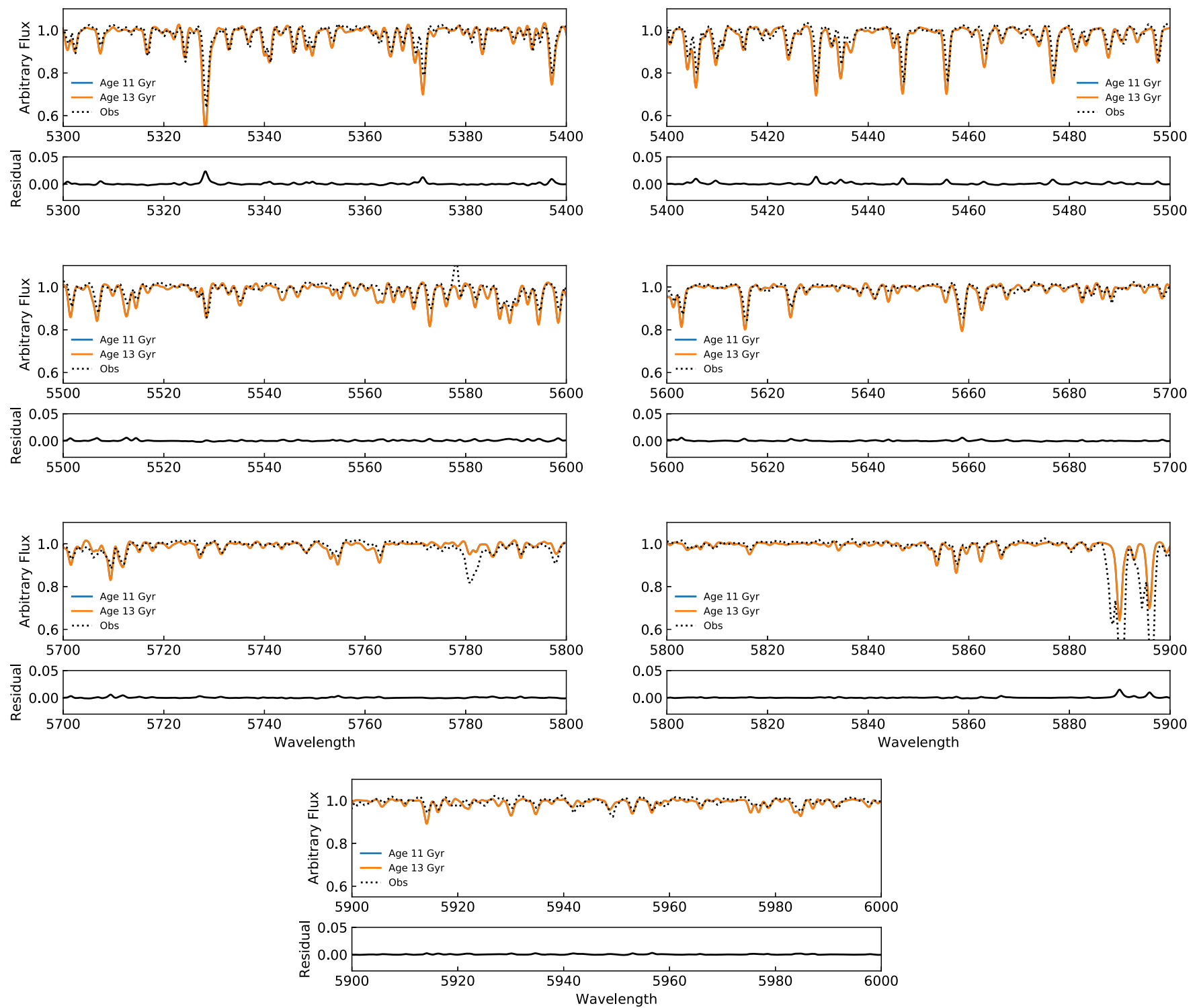

Figure 14. NGC 6522: Synthetic spectra for ages of 11 and 13 Gyr in the region $\lambda \lambda 5300-6000 \AA$, and respective residuals in the lower panels.

Table 7

Na Abundance from the Main Na I Lines in the Integrated Spectra

\begin{tabular}{|c|c|c|c|c|c|c|}
\hline Cluster & $5682.63 \AA$ & $5688.20 \AA$ & $6154.23 \AA$ & $6160.75 \AA$ & $8183.25 \AA$ & $8194.79 \AA$ \\
\hline NGC 6171 & 0.00 & 0.35 & 0.35 & 0.00 & 0.35 & 0.20 \\
\hline NGC 6522 & 0.00 & 0.35 & 0.20 & 0.00 & 0.20 & 0.20 \\
\hline NGC 6624 & 0.20 & 0.35 & 0.35 & 0.35 & 0.00 & 0.20 \\
\hline NGC 6723 & $\ldots$ & 0.35 & 0.35 & 0.35 & 0.20 & 0.00 \\
\hline NGC 6752 & 0.00 & 0.35 & $\ldots$ & 0.35 & 0.20 & 0.35 \\
\hline
\end{tabular}

assuming $Y=0.247,0.257$, and 0.288 , established by Milone et al. (2019), and found ages of 13.4, 13.2, and 13.0 Gyr for $1 \mathrm{G}, 2 \mathrm{G}$, and $3 \mathrm{G}$, respectively.

Therefore, the effects of helium enhancements can be treated in terms of younger ages, as described in the next section.

Effects of age-It is well-known that the effect of age in a CMD is the magnitude of the turn-off point, which is brighter for younger ages. In the integrated spectra there is little change in the lines, given that the RGB stars dominate the flux. This is shown in Figure 14, with the comparison of spectra between 11 and 13 Gyr for NGC 6522, together with the residuals.

\section{Conclusions}

We have built the code SynSSP that combines isochrones for a given age, metallicity, helium abundance, and $[\alpha / \mathrm{Fe}]$, to calculations of a series of synthetic spectra. We are able to 
reproduce the integrated spectra of very old GCs in the metallicity range $-1.6<[\mathrm{Fe} / \mathrm{H}]<-0.7$.

The main aim of this work is to be able to reproduce the integrated spectra of well-known GCs, in order to be able to use this method of building integrated spectra for the analysis of extragalactic GCs, such as those developed by Sakari et al. (2014).

From the present analysis, we can conclude:

1. It is possible to reproduce prominent lines of $\mathrm{Na}, \mathrm{Mg}, \mathrm{Al}$, $\mathrm{Si}, \mathrm{Ca}, \mathrm{Ti}, \mathrm{Ba}$, and $\mathrm{Eu}$, to derive their abundances from the integrated spectra, which are in good agreement with previous results in the literature. This opens the possibility to apply this method to distant star clusters in other galaxies.

2. With the abundances of the most important elements being derived, it is expected that the residual difference between observed and synthetic integrated spectra will be reduced.

3. The effect of age is small among old star clusters. For younger star clusters, they would be distinguishable due to their bluer colors, and by the presence of other sets of lines.
4. The effect of helium enhancement in second (and subsequent) stellar generations is a somewhat younger age, therefore this effect can be neglected.

Finally, a further objective of this work for the future is to use a combination of integrated spectra of SSPs, well-tested with our method, in the building of spectra of galaxies.

T.C.M. acknowledges the post-doctoral FAPESP fellowship No. 2018/03480-7. M.T. thanks the support of CNPq, process number 307675/2018-1. B.B. acknowledges FAPESP project 2014/18100-4 and partial financial support from CAPES Financial project 001 and CNPq. S.R. acknowledge financial support from FAPESP, process number 2015/50374-0 and partial financial support from CAPES Financial project 001 and CNPq.

\section{Appendix \\ Synthetic Spectra-NGC 6522}

The full spectral synthesis performed for NGC 6522 in the spectral range of 4500 to $9000 \AA$ (red lines) are compared to the observed from the WAGGS catalog (Figures 15-19; Usher et al. 2017).
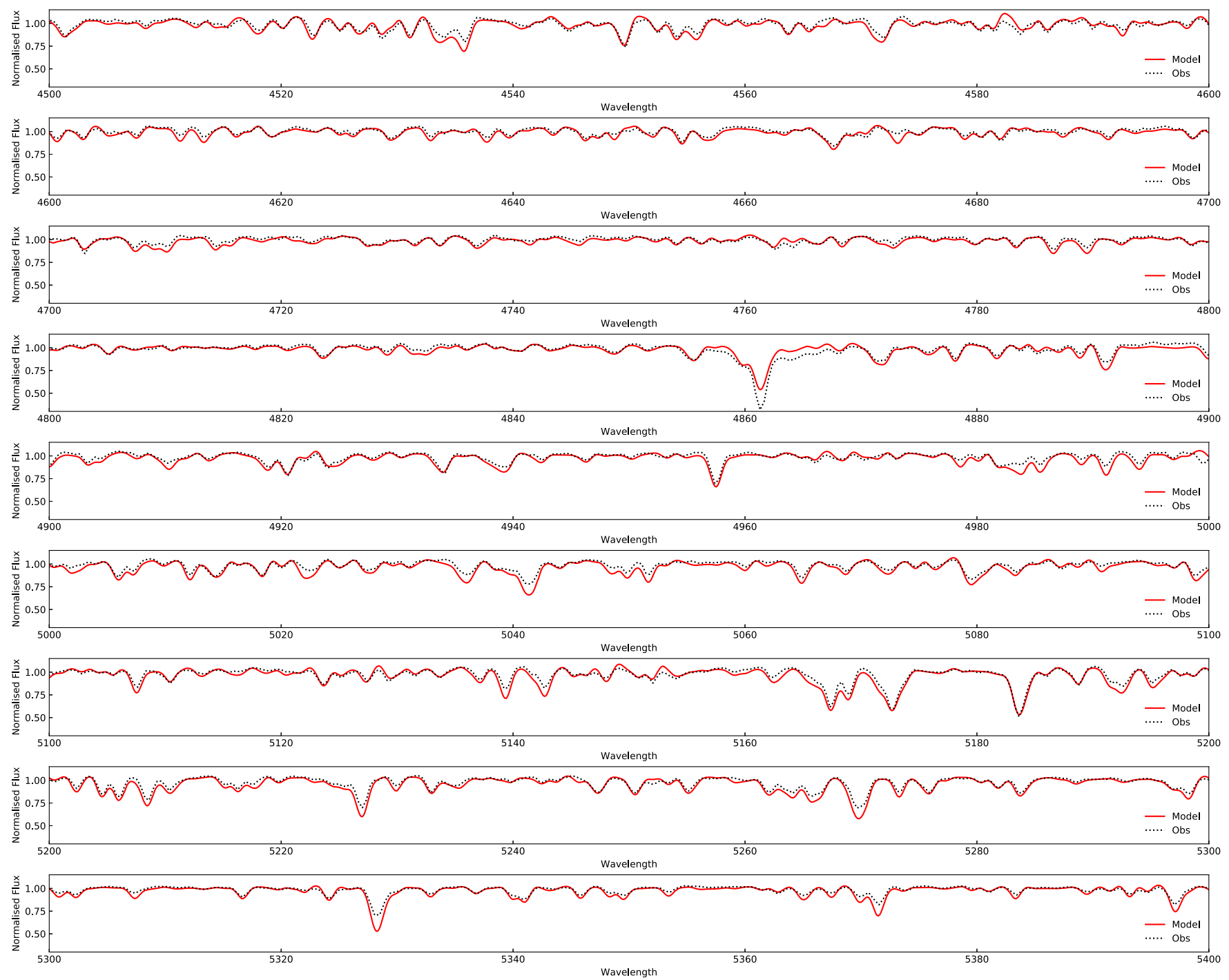

Figure 15. Observed and synthetic spectra for the cluster NGC 6522 in the range 4500-5400 A. Main absorption lines are highlighted. 



Figure 16. Continuation of Figure 15 for spectra in the range 5400-6300 $\AA$. The gray areas represent the region of the telluric line. 

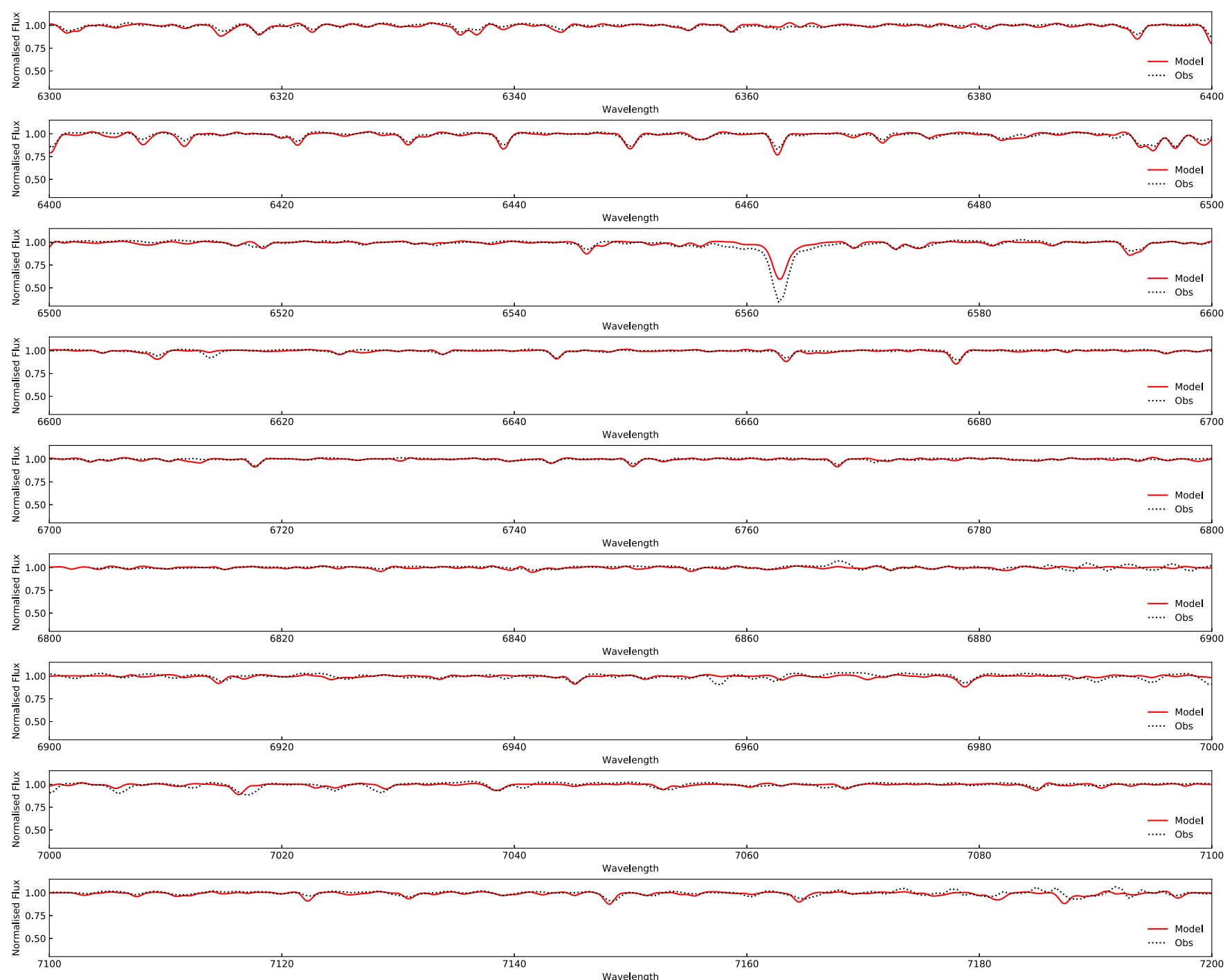

Figure 17. Same as Figure 16 for the wavelength range 6300-7200 $\AA$. 

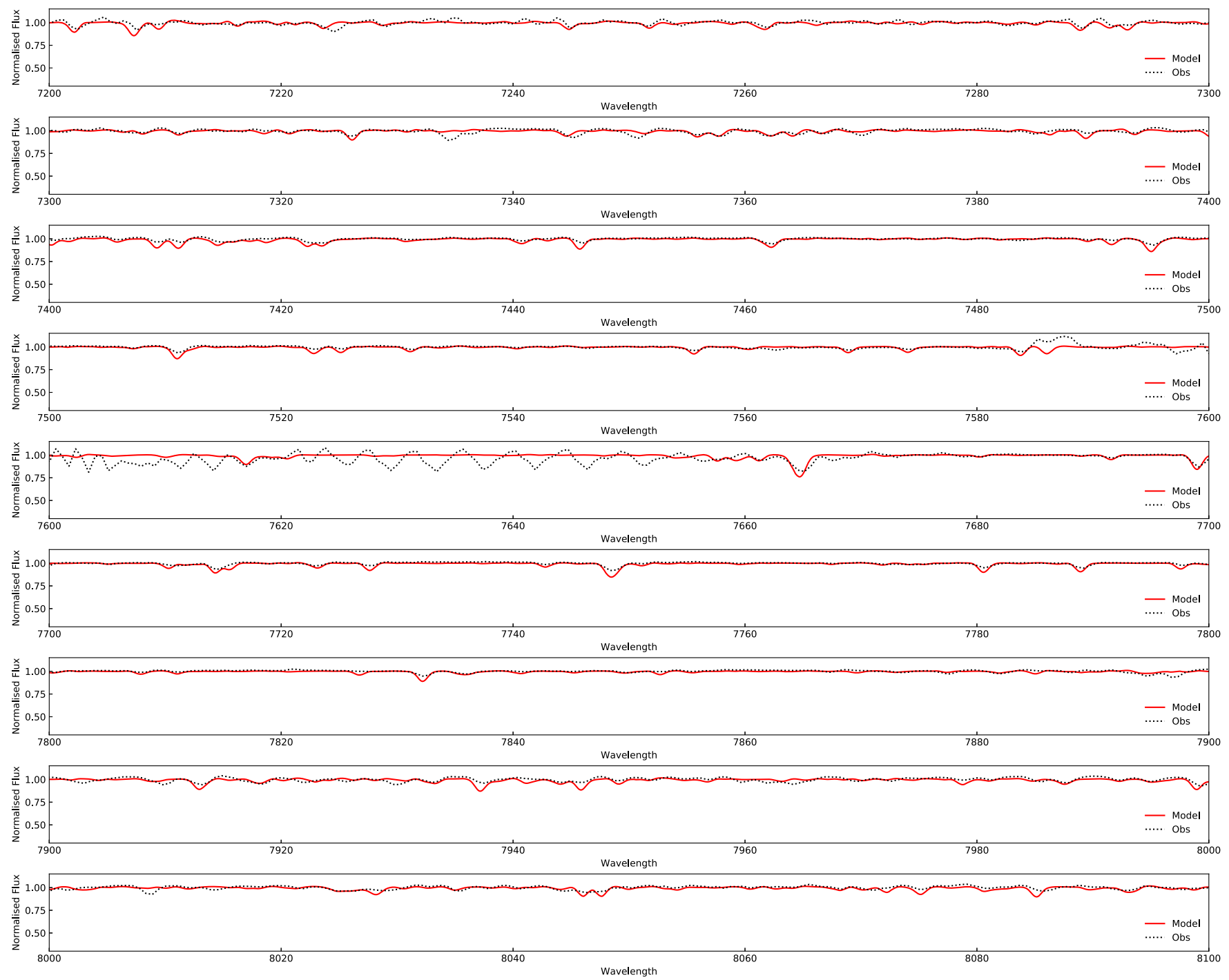

Figure 18. Same as Figure 17 for the wavelength range $7200-8100 \AA$. 

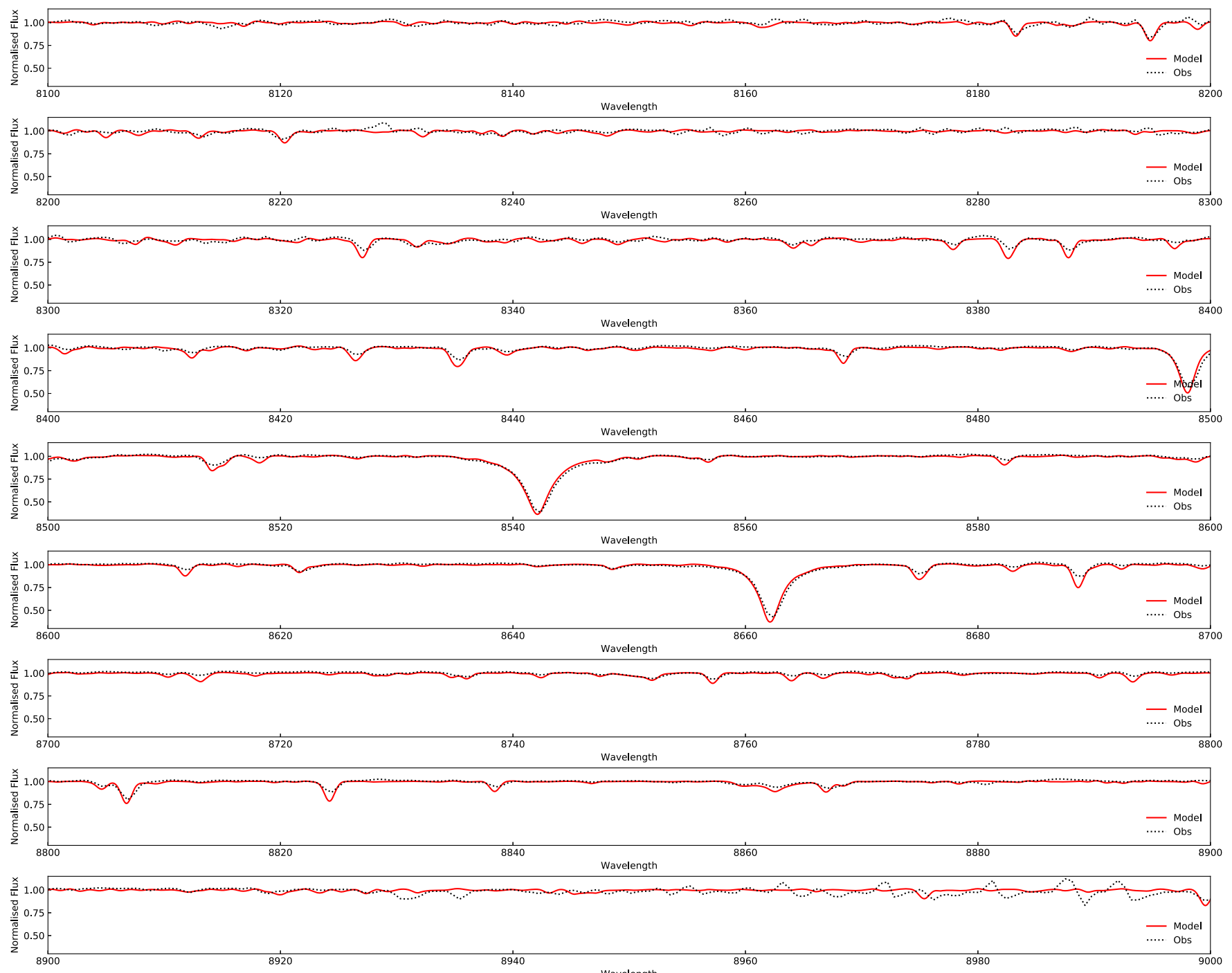

Figure 19. Synthetic and observed spectra for NGC 6522 in the wavelength range $8100-9000 \AA$.

\section{ORCID iDs}

T. C. Moura (i) https://orcid.org/0000-0003-1548-7221

M. Trevisan (iD https://orcid.org/0000-0001-7309-8609

B. Barbuy (i) https://orcid.org/0000-0001-9264-4417

S. Rossi (iD https://orcid.org/0000-0001-7479-5756

\section{References}

Barbuy, B. 1982, PhD thesis, Université de Paris VII

Barbuy, B., Chiappini, C., Cantelli, E., et al. 2014, A\&A, 570, A76

Barbuy, B., Chiappini, C., \& Gerhard, O. 2018a, ARA\&A, 56, 223

Barbuy, B., Perrin, M.-N., Katz, D., et al. 2003, A\&A, 404, 661

Barbuy, B., Trevisan, J., \& de Almeida, A. 2018b, PASA, 35, 46

Bastian, N., \& Lardo, C. 2018, ARA\&A, 56, 83

Bica, E., \& Alloin, D. 1986, A\&A, 162, 21

Bica, E., Ortolani, S., \& Barbuy, B. 2016, PASA, 33, 28

Campbell, S. W., D’Orazi, V., Yong, D., et al. 2013, Natur, 498, 198

Carretta, E. 2019, A\&A, 624, A24

Carretta, E., Bragaglia, A., Gratton, R. G., Lucatello, S., \& D’Orazi, V. 2012, ApJL, 750, L14

Carretta, E., Bragaglia, A., Gratton, R. G., et al. 2009, A\&A, 505, 117

Cayrel, R., Perrin, M.-N., Barbuy, B., \& Buser, R. 1991, A\&A, 247, 108

Chmielewski, Y. 2000, A\&A, 353, 666

Coelho, P., Barbuy, B., Meléndez, J., Schiavon, R. P., \& Castilho, B. V. 2005 , A\&A, 443, 735
Colucci, J. E., Bernstein, R. A., \& McWilliam, A. 2017, ApJ, 834, 105

Conroy, C., \& van Dokkum, P. G. 2012, ApJ, 760, 71

Conroy, C., Villaume, A., van Dokkum, P. G., \& Lind, K. 2018, ApJ, 854, 139

Crestani, J., Alves-Brito, A., Bono, G., Puls, A. A., \& Alonso-Garcia, J. 2019, MNRAS, 487, 5463

Dias, B., Barbuy, B., Saviane, I., et al. 2015, A\&A, 573, A13

Dias, B., Barbuy, B., Saviane, I., et al. 2016, A\&A, 590, A9

Dotter, A., Chaboyer, B., Jevremović, D., et al. 2008, ApJS, 178, 89

Fernández-Trincado, J. G., Zamora, O., Souto, D., et al. 2019, A\&A, 627, 178

Foster, C., Forbes, D. A., Proctor, R. N., et al. 2010, ApJ, 139, 1566

Gratton, R. G., Carretta, E., \& Bragaglia, A. 2012, A\&ARv, 20, 50

Gratton, R. G., Lucatello, S., Sollima, A., et al. 2015, A\&A, 573, A92

Gray, D. F. 2005, The Observation and Analysis of Stellar Photospheres (Cambridge: Cambridge Univ. Press)

Gustafsson, B., Edvardsson, B., Eriksson, K., et al. 2008, A\&A, 486, 951

Harris, W. E. 1996, AJ, 112, 1487

Hinkle, K., Wallace, L., Valenti, J., \& Harmer, D. 2000, Visible and Near Infrared Atlas of the Arcturus Spectrum 3727-9300 A (San Francisco, CA: ASP)

Kerber, L., Nardiello, D., Ortolani, S., et al. 2018, ApJ, 853, 15

Kroupa, P. 2001, MNRAS, 322, 231

Kurúcz, R. L. 2005, MSAIS, 8, 189

La Barbera, F., Ferreras, I., Vazdekis, A., et al. 2013, MNRAS, 433, 3017

La Barbera, F., Vazdekis, A., Ferreras, I., et al. 2016, MNRAS, 457, 1468

Lagioia, E. P., Milone, A. P., Marino, A. F., et al. 2018, MNRAS, 475, 4088

Larsen, S. S., Brodie, J. P., \& Strader, J. 2017, A\&A, 601, A96

Lecureur, A., Hill, V., Zoccali, M., et al. 2007, A\&A, 465, 799 
Lee, J.-W. 2007, RMxAC, 28, 120

Martins, L. P., Lima-Dias, C., Coelho, P. R. T., \& Laganá, T. 2019, MNRAS, 484, 2388

McWilliam, A. 2016, PASA, 33, 40

Meléndez, J., Barbuy, B., Bica, E., et al. 2003, A\&A, 411, 417

Milone, A., Barbuy, B., \& Bica, E. 1995, A\&AS, 113, 547

Milone, A. P., Marino, A. F., Bedin, L. R., et al. 2019, MNRAS, 484, 4046

Milone, A. P., Marino, A. F., Piotto, G., et al. 2013, ApJ, 767, 120

Milone, A. P., Marino, A. F., Renzini, A., et al. 2018, MNRAS, 481, 5098

Milone, A. P., Piotto, G., Renzini, A., et al. 2017, MNRAS, 464, 3636

Ness, M., Asplund, M., \& Casey, A. R. 2014, MNRAS, 445, 2994

Pehlivan Rhodin, A., Hartman, H., Nilsson, H., \& Jönsson, P. 2017, A\&A, 598, A102

Piotto, G., Milone, A. P., Bedin, L. R., et al. 2015, AJ, 149, 91

Pritzl, B. J., Venn, K., \& Irwin, M. 2005, AJ, 130, 2140

Renzini, A., D’Antona, F., Cassisi, S., et al. 2015, MNRAS, 454, 4197

Roediger, J. C., Courteau, S., Graves, G., \& Schiavon, R. P. 2014, ApJS, 210, 10

Rojas-Arriagada, A., Zoccali, M., Vásquez, S., et al. 2016, A\&A, 587, A95

Ryabchikova, T., Piskunov, N., Kurucz, R. L., et al. 2015, PhyS, 90, 054005

Sakari, C. M., Shetrone, M., Venn, K., McWilliam, A., \& Dotter, A. 2013, MNRAS, 434, 358
Sakari, C. M., Venn, K., Shetrone, M., Dotter, A., \& Mackey, D. 2014, MNRAS, 443, 2285

Salpeter, E. E. 1955, ApJ, 121, 161

Sánchez-Blázquez, P., Peletier, R. F., Jiménez-Vicente, J., et al. 2006, MNRAS, 371, 703

Saviane, I., Da Costa, G. S., Held, E. V., et al. 2012, A\&A, 540, A27

Schiavon, R. P., Barbuy, B., \& Singh, P. D. 1997, ApJ, 484, 499

Schiavon, R. P., Rose, J. A., Courteau, S., \& MacArthur, L. 2005, ApJS, 160,163

Smith, G., \& Drake, J. J. 1988, MNRAS, 231, 115

Tenorio-Tagle, G., Silich, S., Palous̃, J., Muñoz-Tuñón, C., \& Wünsch, R. 2019, ApJ, 879, 58

Trager, S. C., Worthey, G., Faber, S. M., Burstein, D., \& González, J. J. 1998 ApJS, 116, 1

Usher, C., Beckwith, T., Bellstedt, S., et al. 2019, MNRAS, 482, 1275

Usher, C., Forbes, D. A., Brodie, J., et al. 2012, MNRAS, 426, 1475

Usher, C., Pastorello, N., Bellstedt, S., et al. 2017, MNRAS, 468, 3828

Valenti, E., Origlia, L., \& Rich, R. M. 2011, MNRAS, 414, 2690

VandenBerg, D. A., Brogaard, K., Leaman, R., \& Casagrande, L. 2013, ApJ, 775,134

Vásquez, S., Saviane, I., Held, E. V., et al. 2018, A\&A, 619, A13

Wegg, C., Gerhard, O., \& Portail, M. 2017, ApJL, 843, L5 